\title{
A numerical framework for constraining synthetic wind fields with lidar measurements for improved load simulations
}

Pettas, Vasilis; Garcia, Francisco Costa; Kretschmer, Matthias; Rinker, Jennifer M.; Clifton, Andrew; Cheng, Po Wen

\section{Published in:}

Proceedings of AIAA Scitech 2020 Forum

Link to article, DOI:

10.2514/6.2020-0993

Publication date:

2020

Document Version

Publisher's PDF, also known as Version of record

Link back to DTU Orbit

Citation (APA):

Pettas, V., Garcia, F. C., Kretschmer, M., Rinker, J. M., Clifton, A., \& Cheng, P. W. (2020). A numerical framework for constraining synthetic wind fields with lidar measurements for improved load simulations. In Proceedings of AIAA Scitech 2020 Forum [1905] Aerospace Research Central (ARC). https://doi.org/10.2514/6.2020-0993

\section{General rights}

Copyright and moral rights for the publications made accessible in the public portal are retained by the authors and/or other copyright owners and it is a condition of accessing publications that users recognise and abide by the legal requirements associated with these rights.

- Users may download and print one copy of any publication from the public portal for the purpose of private study or research.

- You may not further distribute the material or use it for any profit-making activity or commercial gain

- You may freely distribute the URL identifying the publication in the public portal 
See discussions, stats, and author profiles for this publication at: https://www.researchgate.net/publication/338921741

\section{A numerical framework for constraining synthetic wind fields with lidar measurements for improved load simulations}

Preprint · January 2020

CITATIONS

0

7 authors, including:

Vasilis Pettas

Universität Stuttgart

9 PUBLICATIONS 9 CITATIONS

SEE PROFILE

Matthias Kretschmer

Universität Stuttgart

9 PUBLICATIONS 10 CITATIONS

SEE PROFILE

Some of the authors of this publication are also working on these related projects:

Wind Turbine Power Performance View project

Wind Resource Measurements and Modeling View project
READS

144

Francisco Costa García

Universität Stuttgart

2 PUBLICATIONS OCITATIONS

SEE PROFILE

Jennifer Marie Rinker

Technical University of Denmark

16 PUBLICATIONS 43 CITATIONS

SEE PROFILE 


\title{
A numerical framework for constraining synthetic wind fields with lidar measurements for improved load simulations
}

\author{
Vasilis Pettas* \\ Stuttgart Wind Energy (SWE), Allmandring 5b, 70569 Stuttgart, Germany \\ Francisco Costa García ${ }^{\dagger}$ \\ University of Valencia, Av. de Blasco Ibáñez, 13, 46010 València, Spain \\ Matthias Kretschmer* \\ Stuttgart Wind Energy (SWE), Allmandring 5b, 70569 Stuttgart, Germany \\ Jennifer M. Rinker ${ }^{\S}$ \\ DTU Wind Energy, Frederiksborgvej 399, 4000 Roskilde Denmark \\ Andrew Clifton ${ }^{\mathbb{I}}$ \\ WindForS research cluster, Allmandring 5b, 70569 Stuttgart, Germany \\ Po Wen Cheng" \\ Stuttgart Wind Energy (SWE), Allmandring 5b, 70569 Stuttgart, Germany
}

\begin{abstract}
Remote wind sensing technologies, such as lidar, are becoming more and more mature and the wind energy industry is rapidly adopting them for a variety of purposes. One of these use cases is utilizing lidar measurements from a nacelle mounted device in order to improve the accuracy of load simulations by creating more realistic synthetic wind inputs. In this work we present an open source numerical framework, called ViConDAR for "Virtual Constrained turbulence and liDAR measurements", used for simulating lidar measurements and applying them as constraints in synthetic wind field generation. A realistic lidar simulator is used to obtain the virtual lidar measurements by scanning a synthetic wind field. These measurements are fed to open source constrained turbulence generation codes (TurbSim and PyConTurb), coupled to ViConDAR. The resulting constrained wind fields are compared to the original ones in order to quantify the level of convergence and can be used directly as inputs to aeroelastic simulations. Finally, two indicative applications of this framework are shown. First, a sensitivity analysis of the lidar parameters versus varying atmospheric conditions is carried out to investigate the potential of the lidar measurements to capture the wind field properties. Secondly, a sensitivity analysis is presented on the influence of different lidar parameters on the convergence of the full wind fields comparing both turbulence generation codes under varying atmospheric conditions.
\end{abstract}

\section{Introduction}

T IDAR is a remote sensing device used to measure wind speeds along its line of sight (LOS). The main advantages of it, Lcompared to using a meteorological mast for wind energy applications, are 1) its ability to measure speeds remotely over a large range of directions and distances, 2) portability, and 3) cost. Its main limitations are 1) availability, which depends on atmospheric conditions and the sophisticated hardware and software involved, and 2) the uncertainty of measurements due to the requirement for wind field reconstruction (since it measures on a single direction) - especially in complex terrain-, and volume averaging.

\footnotetext{
*Research assistant, University of Stuttgart, Stuttgart Wind Energy (SWE), Allmandring 5b, 70569 Stuttgart, Germany

M.Sc. student,University of Valencia, Department of Earth Physics and Thermodynamics, Av. de Blasco Ibáñez, 13, 46010 València, Spain

${ }^{\ddagger}$ Research assistant, University of Stuttgart, Stuttgart Wind Energy (SWE), Allmandring 5b, 70569 Stuttgart, Germany

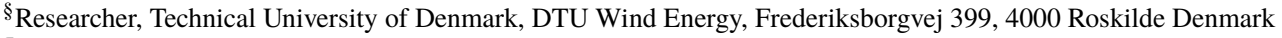

IIManaging director, WindForS research cluster, Allmandring 5b, 70569 Stuttgart, Germany

" Professor, University of Stuttgart, Stuttgart Wind Energy (SWE), Allmandring 5b, 70569 Stuttgart, Germany
} 
In wind energy the main applications are resource assessment [1], power performance testing [2], lidar-assisted turbine control [3] and load validation/verification [4]. A comprehensive analysis of wind energy related lidar applications along with their benefits and limitations can be found in [5].

In this context, we developed an open source, freely available, modular numerical framework called ViConDAR for "Virtual Constrained turbulence and liDAR measurements". ViConDAR is intended to be used by the wind energy community for the different lidar applications with an emphasis on, but not restricted to, constraining turbulent wind fields with lidar measurements using a nacelle mounted lidar for aeroelastic simulations. It can provide realistic virtual lidar measurements of a synthetic turbulence box with varying lidar device properties. The virtual measurements can then be processed to obtain various information about the measured wind field, which can be directly compared with the reference values. Moreover, the measurements can be formatted in the specific input for turbulence generation codes and obtain constrained wind fields in a global format, enabling direct comparisons. The obtained wind fields can subsequently be formatted to the specific input format for aeroelastic codes and be used to perform time domain simulations and compare the response of the turbine between the reference and the constrained inputs. Batch pre and post processing capabilities are also available for all the modules. Further details on the models used and the framework's capabilities are given in section II.

The framework can be used for different applications allowing to easily integrate different methods or interface external codes. To our knowledge this is the only lidar simulator openly available and can be used as a common basis for any academic or industrial application. It can be used in research as a test bench to test new methodologies like: wind field reconstruction, calculation of wind field parameters from measurements, lidar trajectory optimization for different applications, testing constrained turbulence algorithms and perform load analysis with constrained turbulence. Furthermore, it can be used for design optimization of lidar devices for specific applications as well as a tool to assess the lidar requirements and parameters when designing a measuring campaign. Finally, it can be used to perform sensitivity analysis to quantify lidar measurement uncertainties and identify major drivers of uncertainty per application.

In the present study we focus on the load verification/validation cases. In order to reduce the uncertainty in the load validation of an aeroelastic model compared to a real machine, wind input can be improved to better match the reality. In current practice statistics from met mast measurements are used as sole inputs to produce 3D synthetic wind fields commonly called turbulence boxes. These boxes are generated using frequency domain based methods. Since only mean inputs are used -i.e. mean wind speed, turbulence intensity (TI) and wind shear- the spatial and temporal variations of the wind fields in the time domain are generated by using some random input to initiate phase shifts for all the frequency components considered; commonly referred to as turbulence seeds. However, using a nacelle mounted lidar, it is possible to get time series measurements of the inflow with a user defined spatial discretization. These measurements can then be introduced to the turbulence boxes as temporal and spatial constraints. Thus, the wind seen by the blades at each spanwise section during the simulation is closer to the real wind which in turn, is expected to be projected on load simulations leading to reduced uncertainty in load validation. More details on constraining turbulence methodologies can be found in section III.

The scope of the current work is to introduce to the wind energy community a complete open source tool chain for the aforementioned purposes. Additionally, the methodologies for constraining turbulence used in the open source tools TurbSim [6] and PyConTurb [7] are compared and evaluated for lidar based applications. Two applications of the framework are demonstrated in the results section. In the first we show a sensitivity analysis of the lidar measurements to varying lidar setups and atmospheric conditions. In the second a sensitivity analysis is performed on the level of agreement of the constrained wind fields to the original under varying atmospheric conditions and lidar scan patterns using both constrained turbulence algorithms.

The rest of the paper is arranged as follows: In section II we explain the implementation of the overall ViConDAR framework, the lidar simulator and its capabilities. Section III gives a short description of the constrained turbulence concept and an overview of the specific constraining algorithms implemented in PyConTurb and TurbSim. The results in section IV are divided into two parts, demonstrating the two aforementioned use cases of the framework. Finally, in section V some aspects of the suggested methodologies and analysis are discussed further.

\section{Description of the ViConDAR framework}

ViConDAR is an open source modular framework that can be used for different applications of lidar in wind energy. It consists of different modules with different functionalities that can be used independently or sequentially as a tool chain. A schematic overview of the modules and functionalities is given in figure 1.

The starting point is a baseline reference synthetic wind field. This is what we will refer to, throughout the paper, as 
original wind field. The original wind field can be created, for instance, with the Veers [8] or the Mann [9] method with any external code like TurbSim, PyConTurb or the Mann turbulence generator. The files are translated to a global file structure kept throughout ViConDAR. The lidar simulator scans the wind field and provides virtual lidar measurements as time series outputs. More details on the lidar simulator are given in the next section. These virtual measurements can be post processed and provide data, such as wind shear estimation time series, that can be directly compared with each other or the original wind field.

The next step in the tool chain is translating the virtual measurements to the suitable input format for a constrained turbulence generator code, currently TurbSim and PyConTurb. These external codes are running in an automated manner called by the Matlab wrapper. The outputs of the external codes are subsequently converted back to the global wind field format and be compared in order to quantify their convergence using various metrics and error estimates. These outputs of the external codes will be referred to throughout the paper as constrained wind fields. Finally, all wind fields can be translated from the internal global format to wind inputs for aeroelastic simulators (currently FAST [10] and Bladed). The outputs of the time domain aeroelastic simulations can also be post processed through ViConDAR to compare the wind turbine response using the original and constrained wind fields.

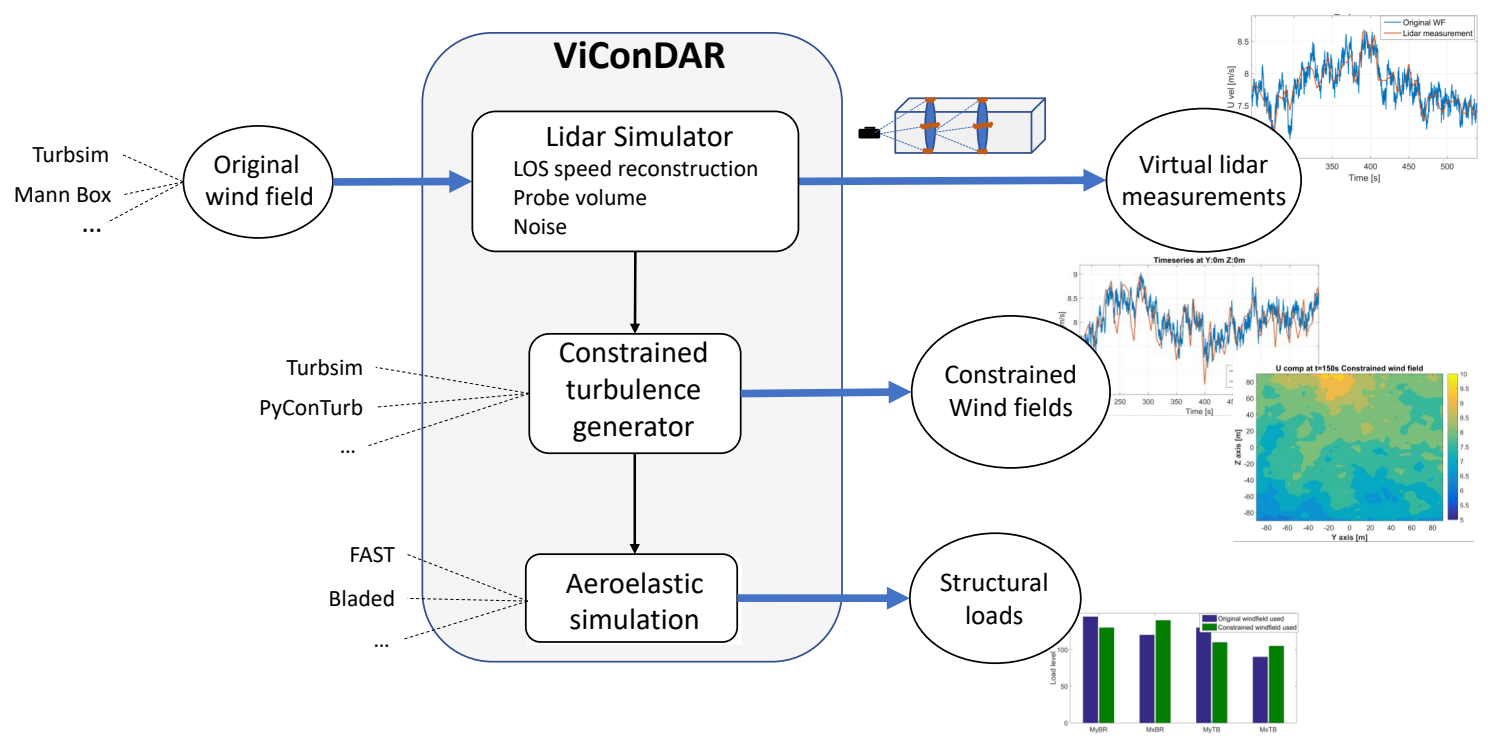

Fig. 1 Overview of the ViConDAR workflow.

As noted earlier, there are different approaches suggested in literature for all the parts of the presented workflow. Nevertheless, only a small part of them are available as open source tools to the wind energy community. We believe that this relatively new topic of research will be relevant in the near future and an open source framework readily available to the community can boost relevant research. Hence, here we only use and develop tools that are offered as open source. The toolbox is developed in a modular manner, so that different users can easily add their modification/method to a specific part like e.g. different wind field reconstruction models or incorporating inputs and outputs to the required input format for another aeroelastic code.

Most of ViConDAR is written in Matlab. Some scripts are also written in python to drive the simulations with PyConTurb. The communication with the coupled external codes is done through text files created to feed the inputs to PyConTurb and TurbSim. The internal wind field format is global for all modules and specific scripts are used to convert from and to this format according to the specific requirements of the module. The user input to ViConDAR is through a single parameter script which is read by the wrapper, driving all the modules. The automated batch processing capabilities are available for all modules and work on a file name basis, which needs to follow a strict user-defined naming convention. More information on the implementation aspects can be found in the code repository.

The ViConDAR framework is offered by the Stuttgart Wind Energy (SWE) institute of University of Stuttgart and can be found at https://github.com/SWE-UniStuttgart/ViConDAR. PyConTurb, developed in DTU, can be found in https://gitlab.windenergy.dtu.dk/rink/PyConTurb while TurbSim 2.0 Alpha, developed by NREL, can be found in https://github.com/OpenFAST/openfast/tree/master/modules/TurbSim. Finally it should be noted that some functions regarding conversion of formats are based/modified from the OpenFAST repository by NREL. The wind field format 
(binary .wnd) currently used is the one used by the open source aeroelastic code FAST [10] by NREL and the commercial code Bladed by DNVGL.

\section{A. Lidar Simulator}

In this section we present the lidar simulator part of ViConDAR and its functionalities. The module provides realistic virtual measurements incorporating the uncertainties involved in real lidar measurements. The lidar can scan the wind field sequentially or in a synchronized manner with any arbitrary lidar scanning pattern, and number of points. The lidar device can be positioned at any point of the $\mathrm{YZ}$ plane (rotor plane). Moreover, there is the option to misalign the whole wind field to the yaw and tilt directions with a constant value.

The lidar properties are defined by the user and can be adjusted to any device. The scanning pattern (or single point) is defined in Cartesian coordinates in a reference plane. The time lag between each consecutive measurement point can also be defined. Thus, the resulting time series have shifted time stamps according to the time lag. This time lag is the time step of the measurements and will be referred as $T_{\text {meas }}$ throughout the paper. The scans are repeated for the full length of the turbulence box. This can be visualized thinking that the turbulence box is moving towards the stationary lidar with the mean velocity of the wind field, since the time dimension and X axis coincide. Figure 2 shows a visualization of a 6 point circular pattern scanning a turbulence box with $T_{\text {meas }} 0.5 \mathrm{~s}$ which results to a $3 \mathrm{~s}$ duration for a full scan of the pattern.

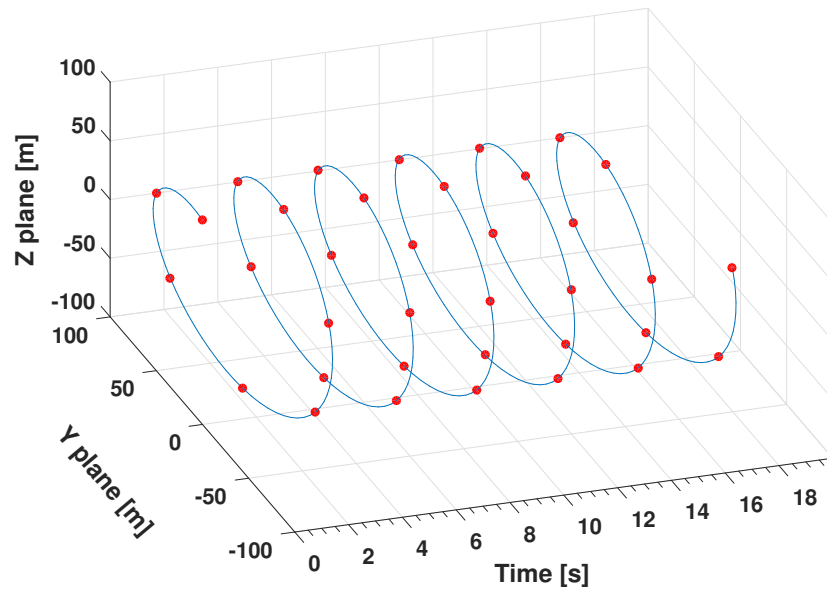

Fig. 2 Example of the spatial and temporal discretization of a circular pattern with seven points and $T_{\text {meas }}=$ $0.5 \mathrm{~s}$.

To imitate both pulsed and continuous wave lidar technologies, a focal distance along the LOS is defined. This distance is the center of the lidar probe volume whose length is user defined. The weighted average of these measurements represents the lidar LOS wind speed measurement at each point of the pattern. The weighting function is user defined, with the current implementations including simple non-weighted averaging and Gaussian distribution centered at the center of the probe volume. Figure 3 shows a 5 point pattern with a probe volume of 60 meters. Each beam takes into account 7 point along the LOS, which are than averaged to give the lidar measurement at the center of the beam's probe volume (purple point in the figure).

In order to obtain the LOS velocity for each pattern point, the $u, v, w$ components of the wind speed in the inertial system are transformed to a LOS coordinate system using the rotation matrices based on azimuth and elevation angles. As lidar can measure speed in the LOS direction only, the velocity component parallel to the LOS direction is kept. Having the 1D LOS lidar measurements, wind field reconstruction methods are needed in order to get the $u_{l i d}, v_{l i d}, w_{l i d}$ measurements, a problem commonly referred to as the cyclops dilemma. There is a variety of approaches suggested in literature to tackle this issue and the research is still ongoing [see e.g. 2]. In the current implementation the reconstruction is done by either assuming that $v$ and $w$ are zero or by assuming that $w$ is zero and a linear correlation between $u$ and $v$. As a result, if there is no misalignment in the flow or no offset of the lidar from the center of the grid the reconstructed $u_{\text {lid }}$ component will match perfectly with the original one. Nevertheless, the combination of probe volume averaging and the LOS speed measurement lead to higher errors as the azimuth and elevation angles increase. In figure 4 we show this by comparing lidar measurements with different probe volume lengths combined with switching on and off the LOS 


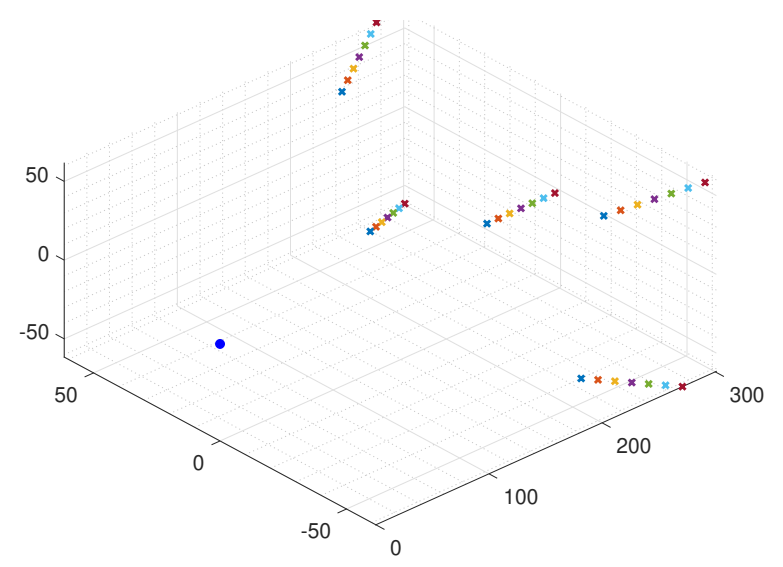

Fig. 3 Example of a 5 point pattern of a square with side length of $100 \mathrm{~m}$ and the center point. Each beam has 7 points along its LOS which are used for representing the volume averaging. The purple point is the probe volume center. The blue dot is the lidar position (the origin). The reference plane is $250 \mathrm{~m}$.

effect.

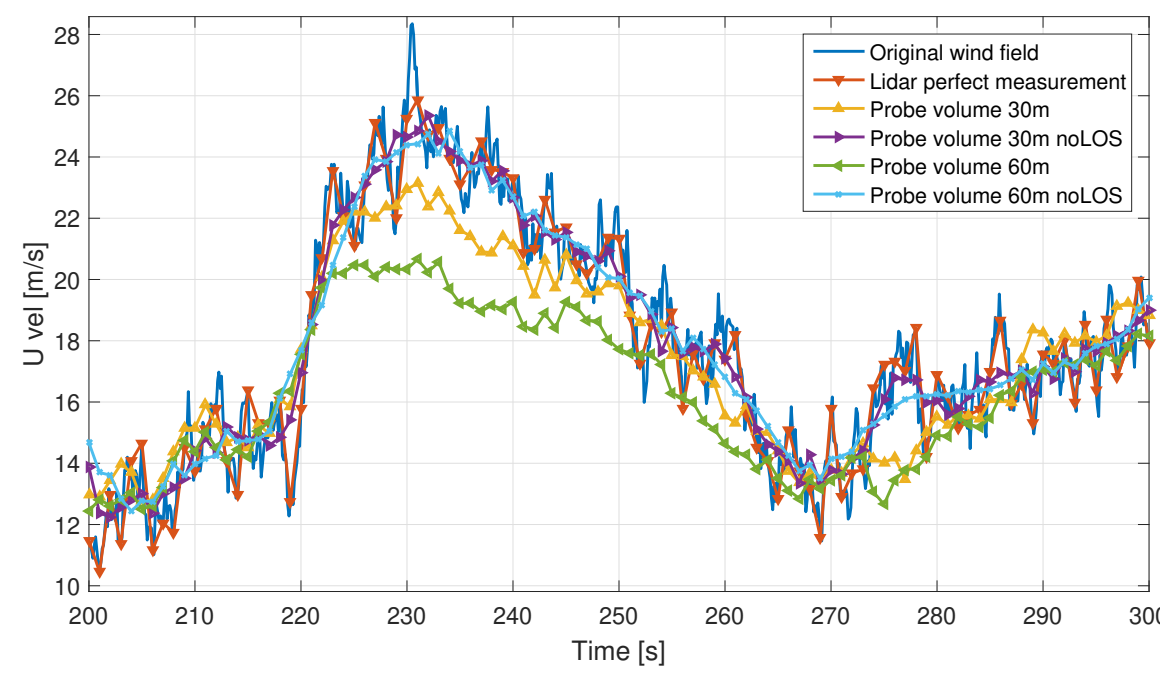

Fig. 4 Effect of probe volume averaging combined with the LOS model on the accuracy of the lidar measurements. The point considered has an elevation angle of $40^{\circ}$ and an azimuth angle of $20^{\circ}$.

The uncertainty of real lidar measurements has multiple sources. These include, among others, the hardware, software and atmospheric conditions. In order to take into account these uncertainties we introduce user defined artificial noise in the measurements. As a first approximation we add Gaussian white noise with a specific signal to noise (SNR) ratio. This a simplistic application and more specific noise models need to be adjusted on a device by device and case by case basis. Figure 5 shows the effect of varying the magnitude of noise (SNR expressed in $\mathrm{dB}$ ) on the virtual lidar measurements.

The obtained virtual lidar measurements can be post processed and compared to the original wind field. In terms of calculated flow characteristics the shear power law exponent is calculated. The shear power law is defined by equation 1.For brevity, in the rest of the paper we will refer to the wind shear power exponent as shear. In order to calculate the instantaneous shear from lidar measurements, but also full wind fields, the following procedure is used: All the points are sorted by height and then averaged in order to keep a single value per height. Then, we fit the power law by minimizing the least square error between the measured points and the points calculated with eq. 1 using the, derivative-free, Nelder-Mead simplex optimization algorithm. As reference height we use the center of the grid and as 


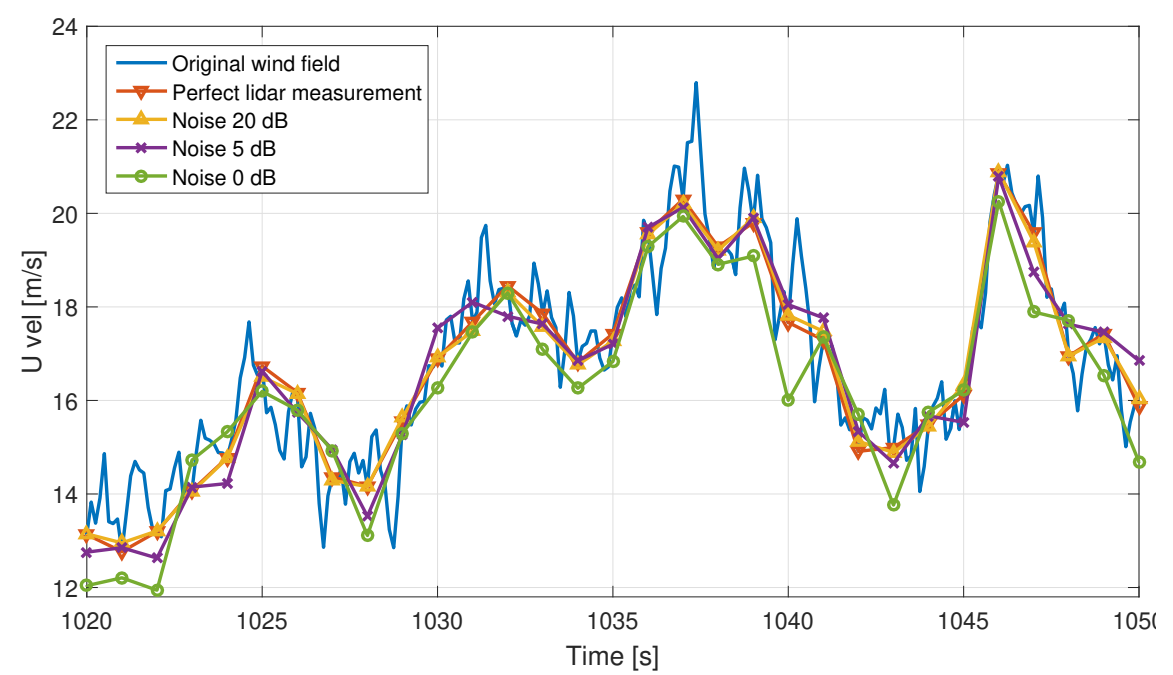

Fig. 5 Effect of added noise to the virtual lidar measurements.

reference wind speed the hub height point (if measured) or the mean of the closest points.

$$
u=u_{\text {ref }}\left(\frac{z}{z_{\text {ref }}}\right)^{\alpha}
$$

Another calculated quantity is the rotor effective wind speed (REWS), defined here as the single wind speed that would produce the same turbine response as the instantaneous turbulent field. Again, in the literature there are a lot of definitions and methods to calculate this quantity for different purposes. The current implementation utilizes a user defined weighting function, based on the distance of the points from the rotor center and takes into account only the points included in the area of the grid covered by the rotor as seen in equation 2 .

$$
R E W S=\frac{\sum_{i=1}^{N} w_{i}\left(r_{i}\right) \cdot u_{i}}{N} \text { with } r_{1 \ldots N} \leq R
$$

The measured time series can be compared against the original wind field in terms of convergence metrics per time series, i.e.: absolute error, root mean square error, variance, turbulence intensity, coefficient of determination and for the whole rotor mean wind speed, REWS and shear. An example of these outputs is presented in figure 6.

\section{Constrained turbulence generation}

In a synthetic turbulence box the grid is defined covering at least the rotor size with each point containing the three dimensional $\vec{u}, \vec{v}$ and $\vec{w}$ components of wind speed. The direction perpendicular to the rotor is also time, since Taylor's frozen turbulence hypothesis is assumed to be valid. This means that there is no wind evolution over time considered, and that the wind field is travelling in the longitudinal direction with the mean wind speed. As also suggested in the relevant certification standards [11], the most commonly used models to generate these synthetic fields are the Mann [9] and the Veers/Sandia [8] models. Based on some predefined coherence spectrum and some mean values, i.e. mean wind speed, shear and TI, the models can generate multiple time domain realizations. These are based on shifting the phases of the different time domain signals, when converting from frequency to time domain, based on randomly defined seeds. In order to reproduce more accurately measured inflows for load simulation and validation purposes, additional input is required. This input can be the time series in multiple points in space as well as coherence spectra if available.

A variety of methodologies can be found in literature for constraining wind fields. Some of the approaches focus on constraining field for specific events. These methods focus more on adding specific properties to the wind fields rather than matching exact measurements and are used in short duration simulations [12, 13] applied on cases like e.g. turbulent operating gusts for extreme load calculation. Other methods based on the Mann model have been applied to longer simulations with full three dimensional wind fields for fatigue load calculations [14-16]. The algorithms of 


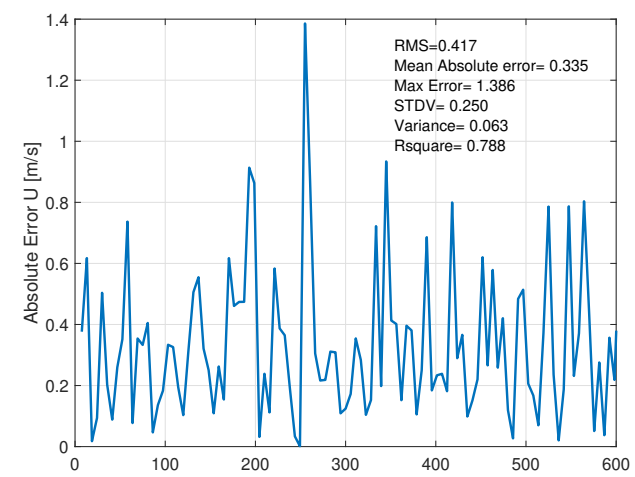

(a)

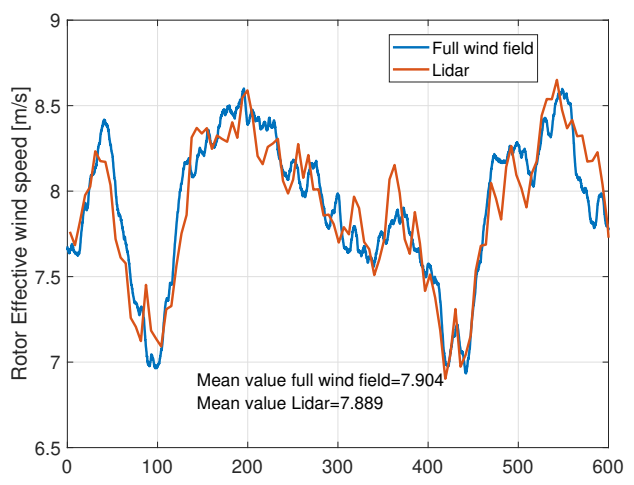

(b)

Fig. 6 Example outputs of the lidar simulator. The same 5 point trajectory as before with 1 sec measurement frequency. Left: absolute error and statistics comparing a lidar point measurement with the same point in the original wind field. Right: Comparison of REWS. The lidar probe volume is considered 30m and added noise SNR is set to $20 \mathrm{~dB}$.

these methods are not available open source. Finally, there are methods based on the Veers models implemented in open source tools $[6,7]$ which are used and shortly introduced here. Both available open source codes for generating constrained turbulence are implemented in the present workflow.

Turbsim is an open source code, written in Fortran and compiled as executable, developed by NREL. It is using the Veers model to create synthetic turbulence. In TurbSim version 2.00 Alpha the functionality to create constrained wind fields was added. It is based on a time series input to calculate frequency domain phase and amplitudes from the zero-mean time series of the measured points. Then, using linear interpolation, in the vertical direction only, the amplitudes for the rest of the points are calculated. The phases of these points, and hence the coherence, are correlated to a single reference point based on a coherence model like e.g. the IEC coherence model. Thus, the coherence between the rest of the measured and generated points is not accounted for. The major challenges with this implementation for the present application are on the one hand that the measurements have to be synchronized which is valid for met mast measurements but not for lidar measurements. On the other hand the 1D interpolation of frequencies and the lack of spatial coherence between the interpolated and measured points reduces the potential benefits of spatially distributed lidar measurements. More information on TurbSim 2.00 Alpha and the underlying models can be found in [6, 17].

PyConTurb is a more flexible, standalone code written in python, closer to the scope of the present application. The code is offered as a python package and is able to generate constrained and random seed-based wind fields. The general approach for creating the wind fields is similar to TurbSim using the Veers model. Despite the similarities the actual algorithm for applying the constraints is different, forcing coherence between all points; measured and simulated. The frequencies of all interpolated points are calculated, as in TurbSim, by linearly interpolating the frequencies of the measured points in the height ( $\mathrm{Z}$ axis) dimension. Here the spatial coherence between the different points is not calculated by the Kaimal spectrum or direct linear interpolation but takes into account each measurement location. The lower-triangular Cholesky decomposition of the covariance matrix is split in two in order to ensure the correct coherence properties taking into account the constraints. This ensures the coherence between measured and simulated points, especially as the distance to the measured point decreases, while preserving the measured time series. More information on PyConTurb and the constraining algorithm can be found in [7]. The input time series have to be synchronous also here, as the method was developed considering met mast measurements.

In current practice the lidar is used as a portable met mast, to derive mean values of shear, TI and mean wind speed which are then used as mean inputs in simulations [see e.g. 18]. These studies show that the lidar is able to capture these mean values with the same or higher accuracy than the met mast. Combined with the decreased cost, portability and increased acceptable wind measurement sectors due to the placement on the nacelle; this is already an important improvement. Nevertheless, the spatial discretization of the lidar can be used to improve further these procedures by including directly the spatial and temporal constraints in the synthetic wind fields. In turn, this is expected to reduce further the uncertainty in applications such as one-to-one load validations. This effect can be understood by looking at figure 7 which shows the same snapshot for an original wind field, a wind field generated with the same mean values but 
different turbulence seed, and two constrained wind fields using lidar measurements from the original with TurbSim and PyConTurb.

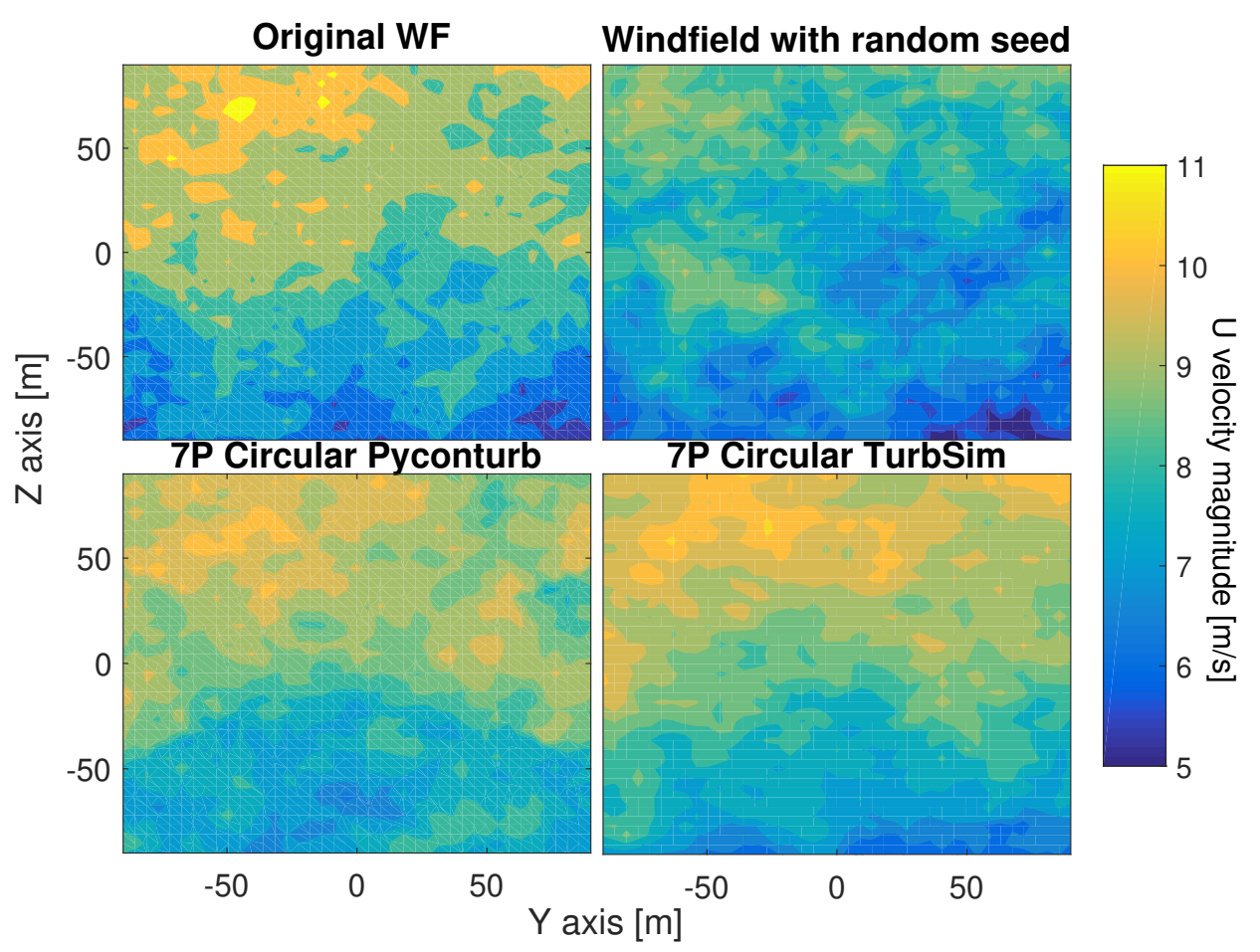

Fig. 7 Instantaneous wind speed (U component) contours of the original, constrained with Pyconturn and TurbSim windfields, and a wind field generated with the same mean values but different random seed.

\section{Results}

The results section is divided in two different applications of ViConDAR. In the first (section IVA) a sensitivity analysis of lidar measurements in respect of the error in calculated wind field characteristics compared to the original wind fields is carried out. The objective is to quantify the expected uncertainty of the lidar measurements under varying conditions. The independent variables considered are: mean wind speed, shear, TI, lidar pattern and measurement sampling frequency. The dependent variables are REWS, $U_{\text {mean }}$ and shear.

In the second analysis (section IVB) we compare constrained wind fields created with TurbSim and PyConTurb with the original wind fields under varying atmospheric conditions. The results intend to quantify the sensitivity of the convergence between constrained and original wind fields to the investigated parameters but also the differences between the two codes used to constrain the wind fields. Here the independent variables are: turbulence generator, shear, $\mathrm{TI}$ and lidar pattern. The dependent variables are: mean absolute $U$ error of all grid points, mean and instantaneous shear, and mean and instantaneous REWS. The REWS is calculated in all cases without applying the weight function in order to avoid biasing the results to a specific weight distribution, i.e. it is the mean of all points lying inside the rotor area. An overview of all the relevant variables for both studies is given in table 1 .

The range of the independent parameter space was chosen in an effort to represent real operational conditions taking into account the IEC standard. The parameters not mentioned here, such as Richardson number and turbulence length scale, were set according to IEC standard. All the original wind fields were created with TurbSim using the Veers method with the IEC coherence model and the Kaimal spectrum. The grid size was chosen as representative of a multi-MW rotor, fitting the DTU 10MW (90m blades) reference wind turbine [19]. The middle of the grid is considered to coincide with the hub height of the turbine at $119 \mathrm{~m}$.

The spatial and temporal discretization of the original turbulence boxes were as dense as possible in order to capture clearly the effects of volume averaging and frequency sampling rate of the lidar while keeping the simulations 
Table 1 Summary of all the lidar parameters and atmospheric conditions considered.

\begin{tabular}{lcc}
\hline & Lidar Measurements & Constrained Wind Fields \\
\hline Umean [m/s] & 8,14 & 8,14 \\
TI [\%] & $5: 5: 30$ & $5: 5: 30$ \\
Shear exponent [-] & $0: 0.05: 0.25$ & $0: 0.05: 0.25$ \\
Surf. Roughness L [m] & 0.03 & 0.03 \\
Seeds & 6 & 1 \\
Lidar pattern & $5 \mathrm{P}, 7 \mathrm{P}, 9 \mathrm{P}$ & $7 \mathrm{P}, 12 \mathrm{P}$ \\
$T_{\text {meas }}[\mathrm{s}]$ & $0.25: 0.25: 2$ & 0 \\
Tpat [s] & $T_{\text {meas }} \times$ NoPoints & 2 \\
Focal distance [m] & 250 & 250 \\
SNR [dB] & 20 & 20 \\
Probe Volume [m] & 30 & 30 \\
Total grid time [s] & 1800 & 1800 \\
Grid dt [s] & 0.125 & 0.125 \\
Grid size [m] & $180 \times 180$ & $180 \times 180$ \\
$\Delta$ grid [m] & 4.5 & 4.5 \\
\hline
\end{tabular}

computationally feasible. The total time of the wind fields and number of random turbulence seeds was set as high as possible in order to make the comparisons statistically independent to the random seed realization. A preliminary convergence study showed that the selected values were sufficient. In the case of the constrained wind field comparison study, only one seed was used due to limitations in computational power although a short study showed that more turbulence seeds were required and it is a known limitation of this study. We discuss this issue in section IV.

All the parameters chosen here do not represent a specific lidar device or wind site. The goal is to perform a generic sensitivity analysis in order to show the potential of such studies using ViConDAR and derive some generalized conclusions about the lidar behavior and the turbulence generators' capabilities. The lidar scan patterns considered in all simulations according to the previous table, are shown in figure 8. The naming convention shown here will be used throughout the result section. The lidar patterns were not chosen based on optimization but based commonly used patterns. The lidar setup imitates a nacelle mounted pulsed lidar, perfectly aligned with the hub center at $[0,0]$, with range gate length of $30 \mathrm{~m}$, and we only use the range gate with center at $250 \mathrm{~m}$ along the LOS. The focal distance was chosen intending to measure at a distance of $2.5 \mathrm{D}$ upstream. We want to be close enough to the turbine, so that the wind evolution does not change significantly the wind seen by the turbine and at the same time far enough to be out of the induction zone. In the first study the lidar is considered a scanning lidar with varying sampling rate. In the second we consider a multi-beam lidar with synchronized measurements. This is done due to the limitation of the turbulence generators to accept time shifted measurements and in order to avoid biasing the result by interpolating or averaging the lidar measurements.

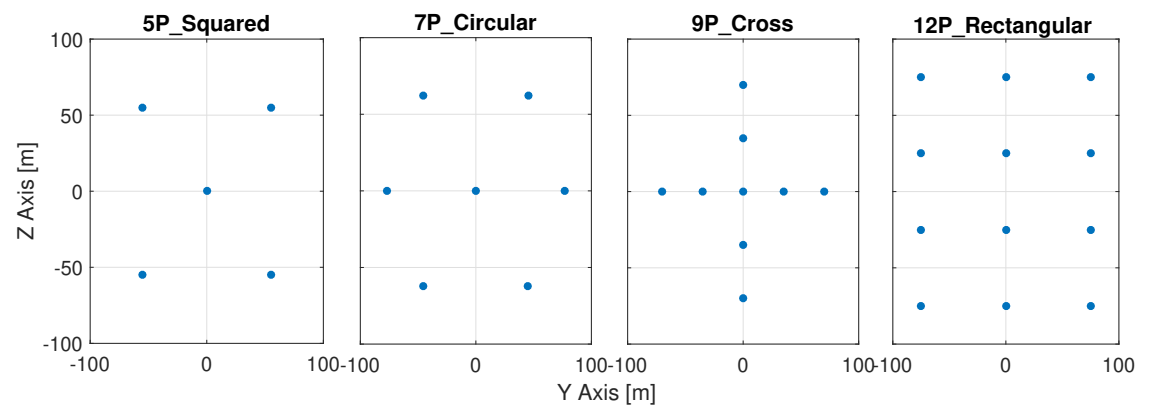

Fig. 8 Illustration of the 4 scanning patterns considered for the comparison study. 
The errors shown in all figures are absolute errors and are calculated in two ways. First, we calculate the mean absolute of the error (MAE) time series (as seen in figure 6a) with equation 3. This is is more relevant for load validation cases, where the instantaneous error can affect the load response of the turbine. Secondly, we calculate the error of the mean of the quantity for the whole wind field using equaiton 4 which is the common practice as e.g. in wind measurement campaigns it is common to use 10 minute averages. Absolute values are used in both cases as we want to use one value to quantify each result. The mean of the error is very close to 0 since there is no systematic positive or negative difference between the compared quantities. The percentages of the errors shown are the absolute errors divided by the original wind field value (mean or instantaneous).

$$
\begin{gathered}
\epsilon_{1}=100 \cdot\left(\frac{\sum_{i=1}^{N} \frac{\left|Q_{\text {Cons }_{i}}-Q_{O r_{i}}\right|}{Q r_{i}}}{N}\right) \quad[\%] \\
\epsilon_{2}=100 \cdot\left(\frac{\left|\overline{Q_{\text {Cons }}}-\overline{Q_{O r}}\right|}{\overline{Q_{O r}}}\right) \quad[\%]
\end{gathered}
$$

\section{A. Sensitivity analysis of lidar measurements in respect to lidar parameters and atmospheric conditions}

The contours in figure 9 show the calculated mean absolute error of the shear time series calculated from the lidar measurements under varying TI and shear conditions with the three considered patterns for a $T_{\text {meas }}$ of $0.25 \mathrm{~s}$. The $7 \mathrm{P}$ pattern shows the lower error in all cases and it seems to be the least sensitive in varying conditions. Although the 9P pattern has the most points in the vertical direction it performs the worst, suggesting that the variation on the lateral direction is important for shear estimations especially when large areas are considered. As expected, the instantaneous shear estimation is more accurate in conditions with low TI and high shear with the error increasing almost proportionally with increasing turbulence and decreasing shear. The effect of TI appears more dominant than the effect of shear in all cases. The magnitude of error, being higher than $50 \%$ and reaching over $100 \%$ in some cases, suggests that the present lidar configurations are not reliable for calculating instantaneous shear after some lower threshold of shear and upper of turbulence. Moreover, the error increases with increasing mean velocity for all patterns but the trend does not change. This was observed in all results, hence for the rest of the comparisons we will show only the $8 \mathrm{~m} / \mathrm{s}$ case. Finally, by examining the results individually (not shown here) there was not a clear trend identified on whether the shear is under- or overestimated.

The MAE of the shear time series with varying $T_{\text {meas }}$ and shear exponent for wind fields with mean speed of $8 \mathrm{~m} / \mathrm{s}$ and TI of $15 \%$ is presented in figure 10 . For shear values lower than 0.15 , the error is inversely proportional for all patterns and time steps. As seen also before, for higher shear values the error is quite small and less sensitive to shear variations. Variation of $T_{\text {meas }}$ for shears up to 0.15 seems to have no affect on the error. For higher values the error increases with $T_{\text {meas }}$. This is explained by the fact that the slower the sampling rate the farther the pattern points are distributed space resulting to average errors compared to the original wind field. For the considered setup it seems that a shear value around 0.15 is the point below which this error becomes more significant. In terms of patterns the 7P shows the lowest error for all conditions with the $9 \mathrm{P}$ showing the highest.

In figure 11 we show the error of the mean shear for the 3 patterns with $T_{\text {meas }} 0.25 \mathrm{~s}$ and varying TI and shear. This error is much lower than the mean error of the time series. This is in agreement with other studies [e.g. see 4] suggesting that the mean values obtained by the lidar are as accurate as the cup or sonic anemometer. As before low turbulence and high shear regions show the least error. The difference here is that this error seems to be far less sensitive to the pattern with all cases showing an error lower than $5 \%$ for conditions with shear higher than 0.15 and turbulence lower than $15 \%$.

The MAE of the REWS time series for varying $T_{\text {meas }}$ and TI is shown in figure 12 . The shear is 0.15 and mean wind speed $8 \mathrm{~m} / \mathrm{s}$ for all cases. Here the error is much smaller compared to the shear. The time step shows also here a minimal effect on the error, even in high turbulence conditions. The error increases proportionally with TI in a similar rate for all patterns. The lowest errors are found at the 7P pattern but the differences are marginal and do not allow for identifying clearly an optimal pattern. The same trends were identified with the other shear cases considered (not shown here).

Figure 13 shows the MAE of the REWS time series for varying shear and TI conditions for a mean speed of $8 \mathrm{~m} / \mathrm{s}$ and a $T_{\text {meas }}$ of $0.25 \mathrm{~s}$. As in the previous case the REWS error seems to be sensitive only to the TI level and not to the shear variation. The level of error is lower than $5 \%$ in all cases with marginal differences lower than $1 \%$ for all patterns. For the $14 \mathrm{~m} / \mathrm{s}$ average wind speed fields the results were quite similar with the maximum error approaching $7 \%$. 


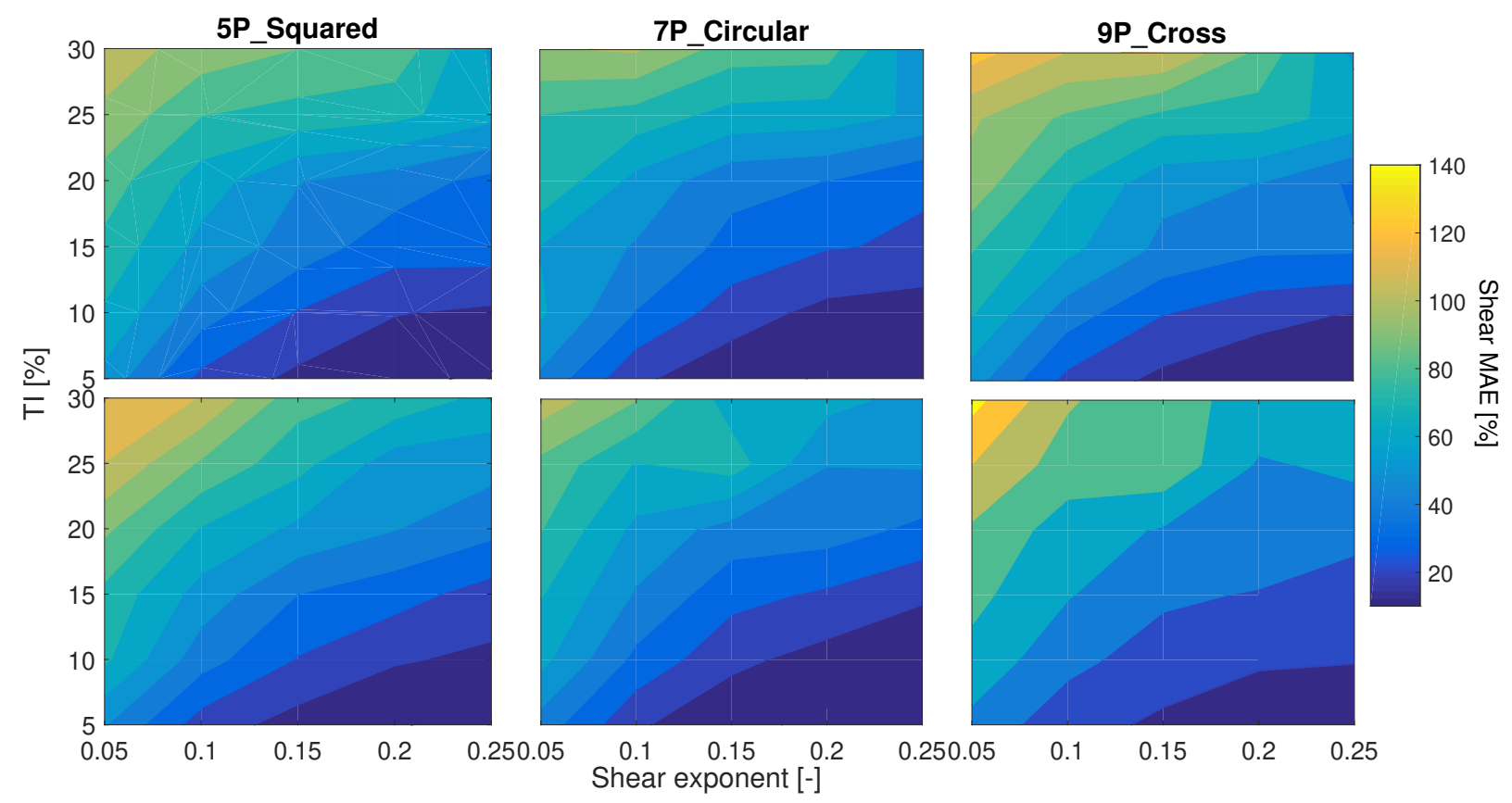

Fig. 9 MAE percentage of the shear time series for the 3 patterns with a $T_{\text {meas }}$ of $0.25 \mathrm{~s}$. Upper row mean velocity $8 \mathrm{~m} / \mathrm{s}$ and lower $14 \mathrm{~m} / \mathrm{s}$ for wind fields with varying TI and shear.

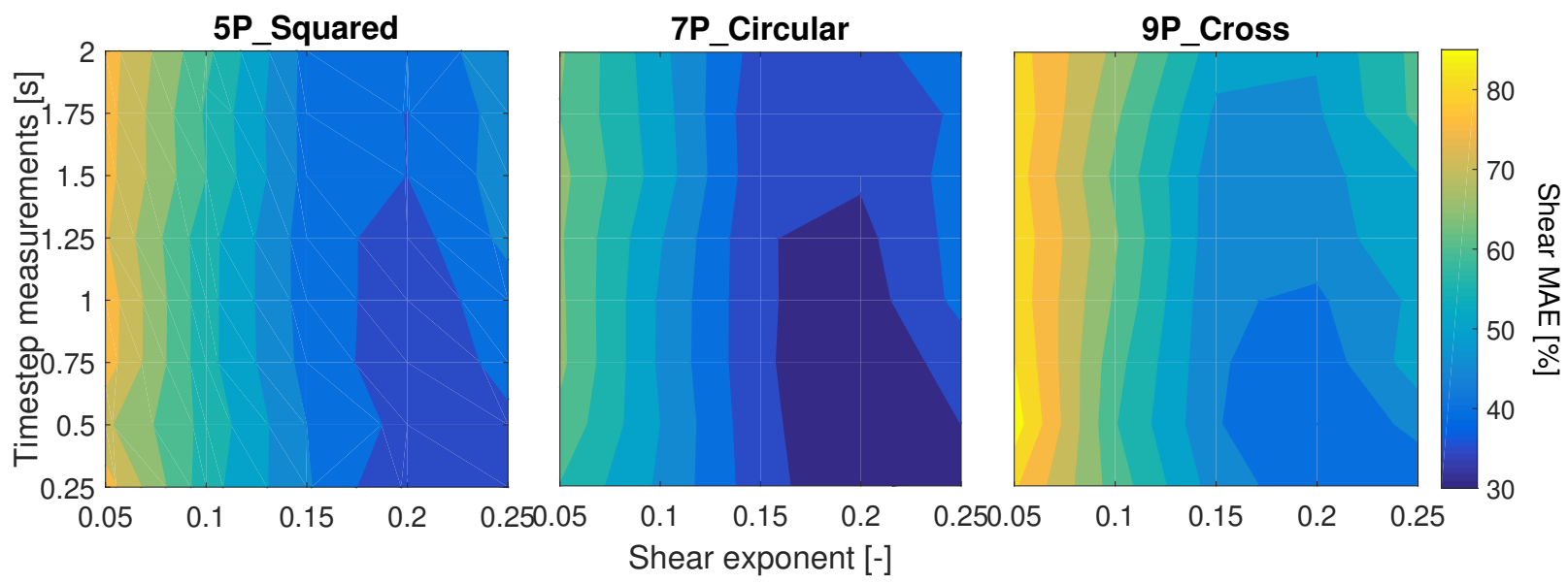

Fig. 10 MAE percentage of the shear time series for the 3 patterns with varying shear and time step of measurements. Turbulence intensity is $15 \%$ and mean wind speed $8 \mathrm{~m} / \mathrm{s}$.

Finally, the results for the error of the mean REWS are not shown here since the error was lower than $1 \%$ for all cases. This is expected since the mean value of the velocity depends mainly on the error of the measurement rather the pattern or the conditions. The errors correlated with mean speed are expected to increase if yaw misalignment (varying or constant) of the lidar to the wind field is introduced.

\section{B. Comparison of constrained methodologies under different atmospheric conditions}

In this section we compare the constrained wind fields to the original ones. Initially, the difference between a measured point and an interpolated point by the constrained turbulence algorithm is shown in figure 14. The plot shows the $U$ component wind speed time series of a point in the grid which is measured when using the 7P pattern, and interpolated when using the $12 \mathrm{P}$ pattern. Both TurbSim and PyConTurb apply the input constrains at the measured points. There is a small offset between the two codes which can be attributed to different calculation of the shear from 


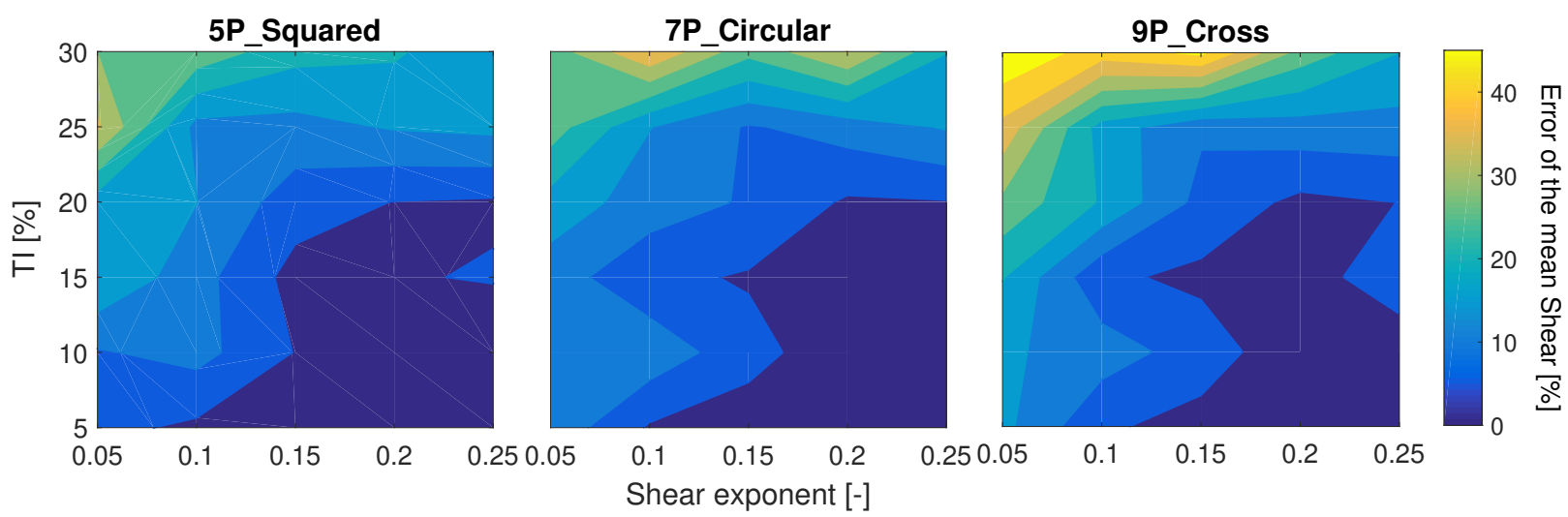

Fig. 11 Absolute error of the mean shear in percentage for the 3 patterns with varying shear and TI with a time step of measurement $0.25 \mathrm{~s}$. Mean velocity is $8 \mathrm{~m} / \mathrm{s}$.

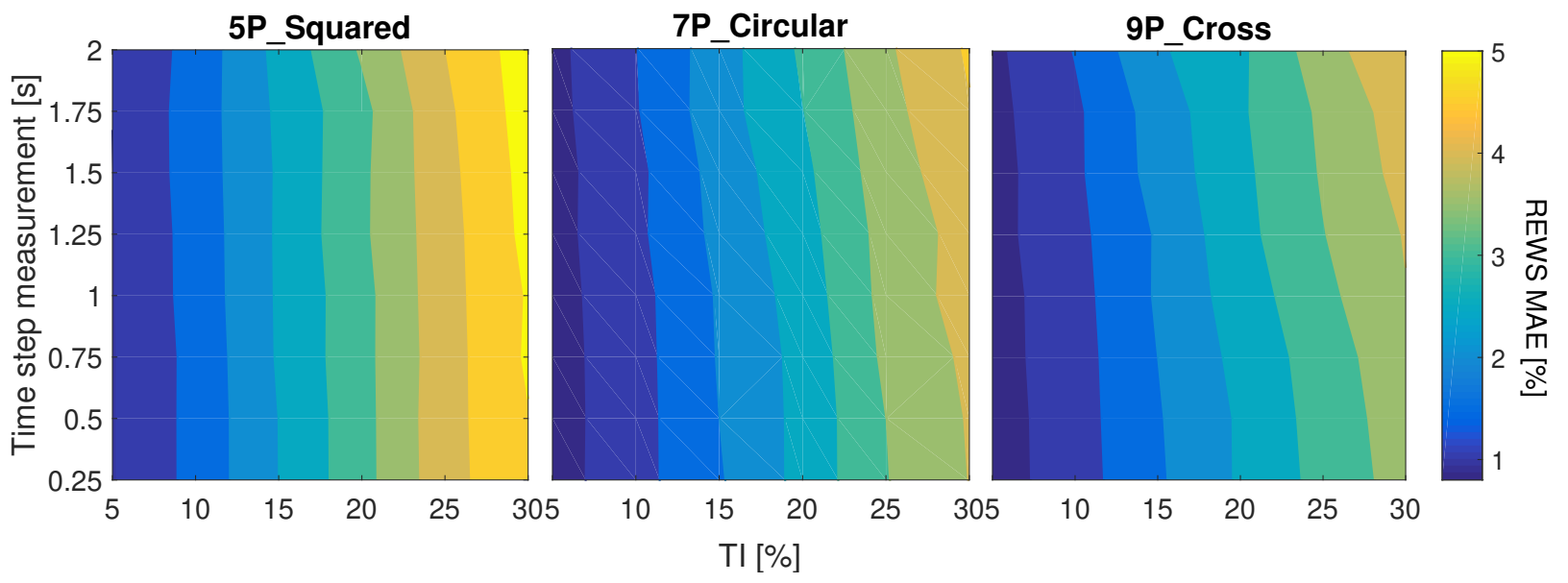

Fig. 12 MAE percentage of REWS time series for the 3 patterns with varying turbulence and time step of measurements. Shear exponent is 0.15 for all cases and mean wind velocity $8 \mathrm{~m} / \mathrm{s}$.

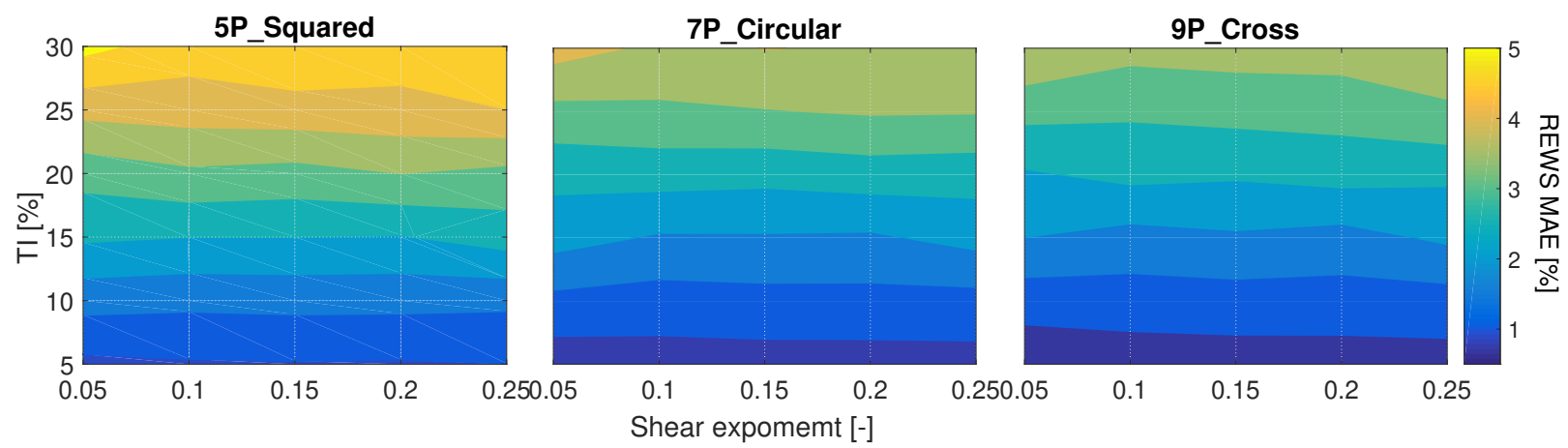

Fig. 13 Mean absolute error percentage of shear time series for the 3 patterns with varying shear and turbulence intensity. Time step of measurements is set to $0.25 \mathrm{~s}$ for all cases and mean wind velocity $8 \mathrm{~m} / \mathrm{s}$.

the measurements. When the point is not measured the time series is interpolated by the neighbouring points based on the frequencies of the constrained points. The phases are then created based on the underlying spatial coherence model.

In figure 15 we show the comparison of the average $U$ wind speed per time slice time series between the constrained and original wind fields with the reference having a shear of 0.15 ant TI $15 \%$. The pattern used for the lidar measurements 


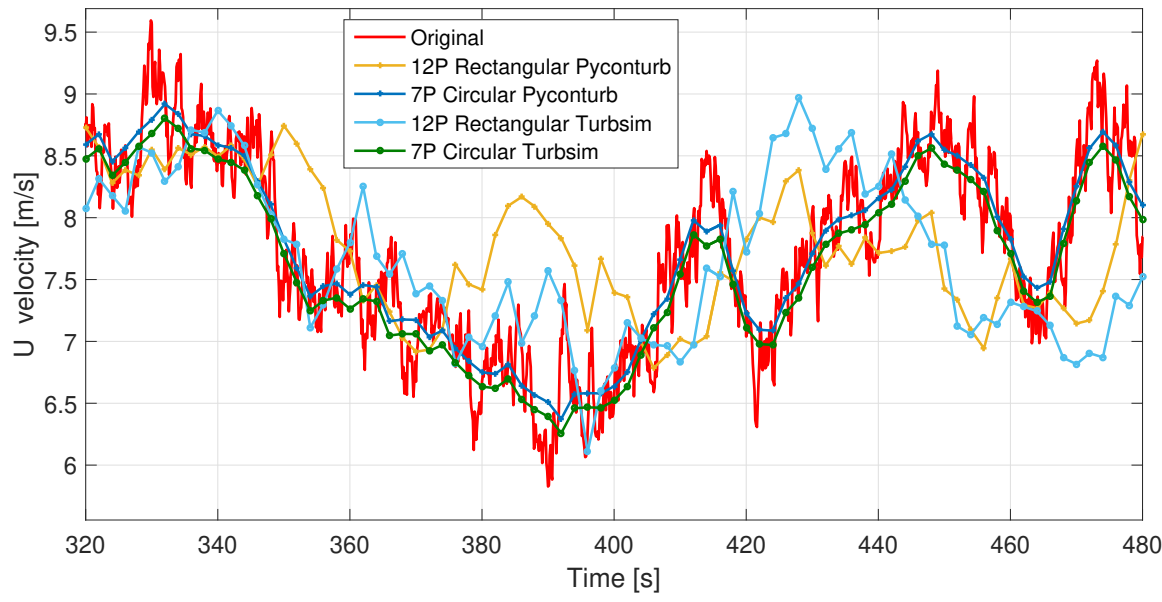

Fig. 14 Time series of $U$ wind speed at grid point $Y=45 \mathrm{~m}, Z=64 \mathrm{~m}$ for original and constrained wind fields with different patterns. This is a measured point for the 7P Circular pattern while for the 12P rectangular pattern it is interpolated by the constraining algorithms.

is the 7P Circular. The averaging effect does not allow to determine how good the spatial distribution of wind speeds matches, but it is an indicator of how the mean constrained wind field follows the variations of the original one. As we will also see in the following results, the PyConTurb algorithm is able to follow better the variations of the original wind field.

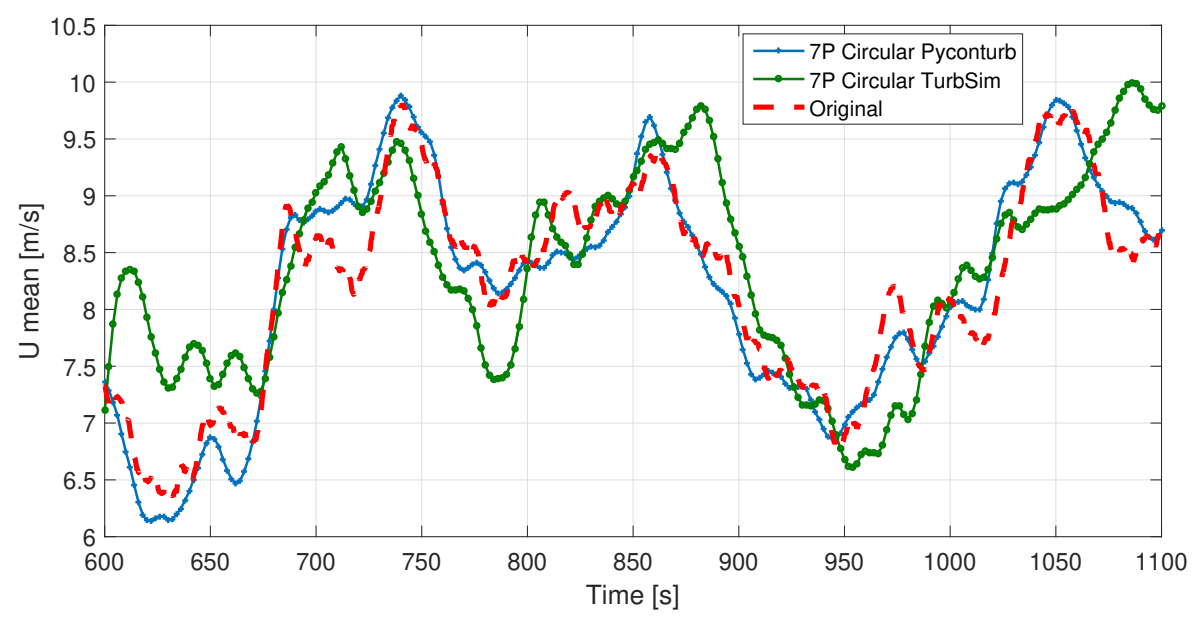

Fig. 15 Time series of the average wind speed per time slice. The original wind field has $8 \mathrm{~m} / \mathrm{s} \mathrm{mean}$ wind speed, shear exponent 0.15 and turbulence intensity $15 \%$. The constrained wind fields are created by the same virtual lidar measurements with the 7 point circular pattern.

The previous observation can be explained by looking at figure 16 which shows the mean absolute error percentage of the $U$ component time series, i.e. the mean of the MAE of each time series divided by the original time series, for all the grid points for the same case as before. The spatial coherence of each non-measured point is calculated in PyConTurb taking into account the closest constraints. The TurbSim algorithm imposes the constraints to the measured points and for the rest of the points calculates the phase based on the spatial coherence with respect to the reference point (in this case the center [0,0]). In the plot we see the error for PyConTurb being lower in the neighborhood of each constraint and increasing as distance from constraints increases. For TurbSim we see that the error is minimal around the center point and increases with distance. The other constrained points have no coherence with their neighbouring points, despite the fact that the error on the measured points is very low.

In figure 17 we show how the previous observation on the spatial discretization of the error translates to the temporal 

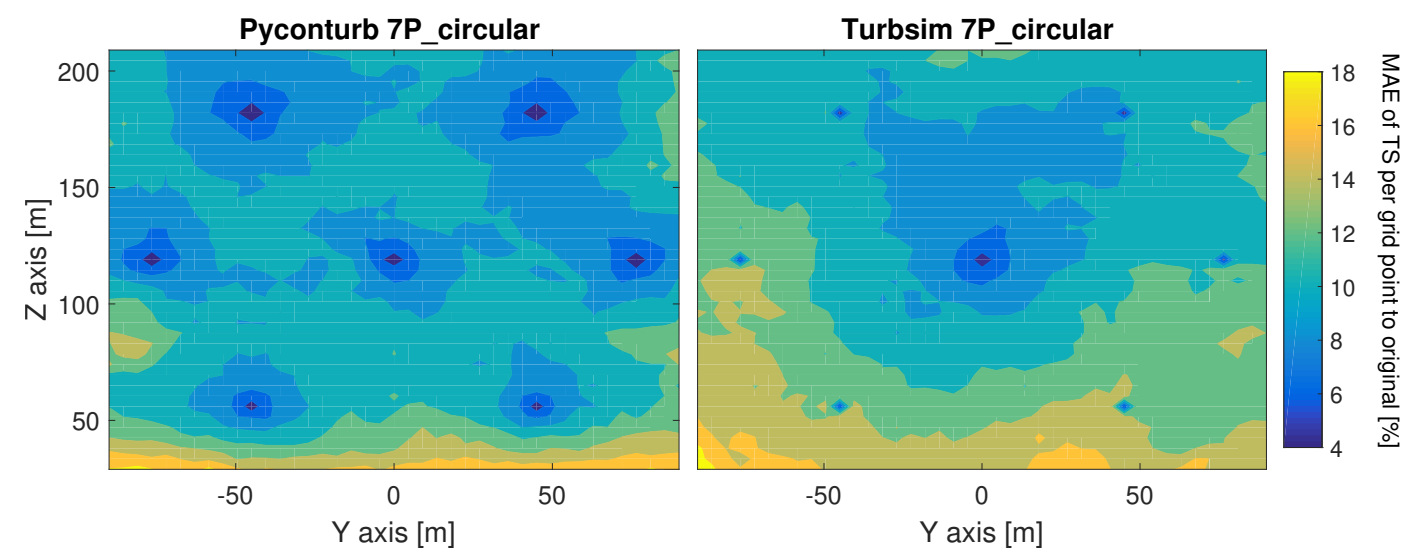

Fig. 16 Contour of mean absolute error percentage of each grid point $U$ time series compared to the original wind field with TurbSim and PyConTurb.

discretization. The instantaneous absolute error percentage of all grid points is averaged in order to obtain the time series of the error. We use this metric, and in the following results the mean of it for each simulation case, in an effort to quantify the spatial and temporal agreement of the wind fields with one value. This metric is not optimal, as we noticed that the result can, in some cases, be biased by outliers where a large error in a single point with very low wind speed can lead to overestimation of the, otherwise significantly lower, error. Combining figures 16, 17 explains why, when using a lidar instead of a metmast, it is important to take into account the 2 dimensional spatial correlation of the constraints to the interpolated points.

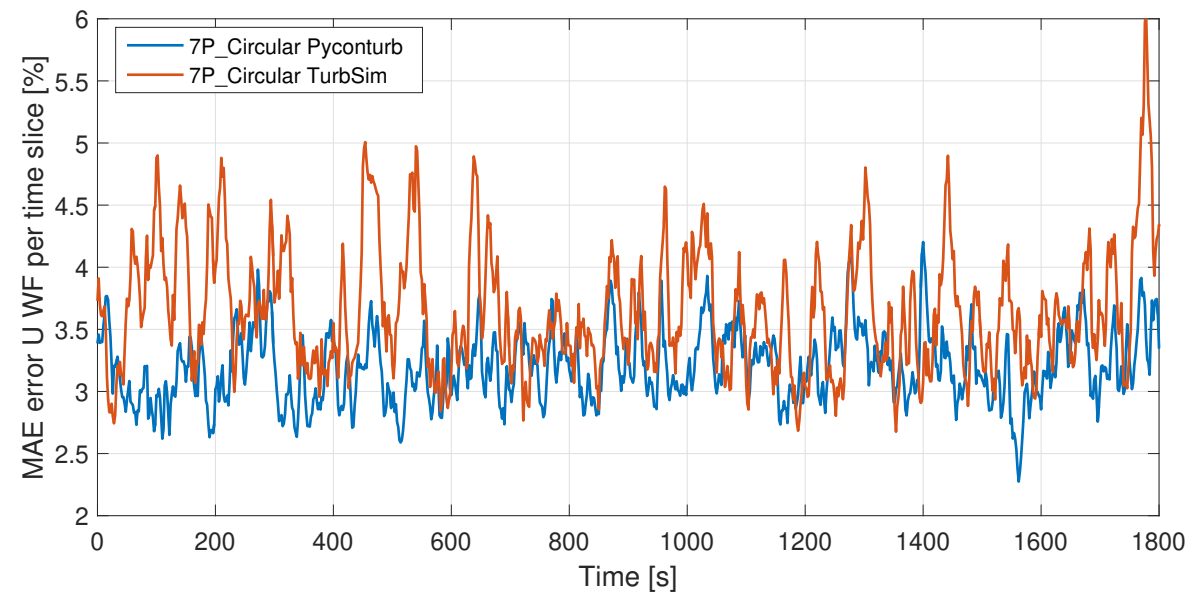

Fig. 17 Times series of the instantaneous mean absolute error percentage of $U$ for the whole grid.

Figure 18 shows the MAE percentage of the shear time series, between constrained and original wind fields, for varying atmospheric conditions using both codes. The mean wind speed is $8 \mathrm{~m} / \mathrm{s}$ and the time step for both used patterns considered is $2 \mathrm{~s}$. The instantaneous shear error calculated with the PyConTurb algorithm is about $50 \%$ lower in most cases. This is explained again by the difference in the spatial coherence method where more points, in the vertical direction, follow the behavior of the original points. The variation according to atmospheric conditions is similar to the results for the lidar measurements where the error increases with decreasing shear and increasing TI. Regarding the patterns, we see that using the 12P pattern reduced, in both cases, the magnitude of the error by $10 \%-20 \%$ while showing the same trend with the $7 \mathrm{P}$ pattern in terms of error distribution along conditions.

The effect of TI and shear variations on the mean absolute error percentage is shown in figure 19. Each point of the contour grid is the mean of the error shown in figure 17. The dominant effect of turbulence is apparent. The magnitude of the error is nearly proportional to TI while it appears not to be influenced by the shear. In terms of algorithm comparison, the trends are very similar with the magnitude of error being lower by $5 \%-10 \%$ with PyConTurb. The 


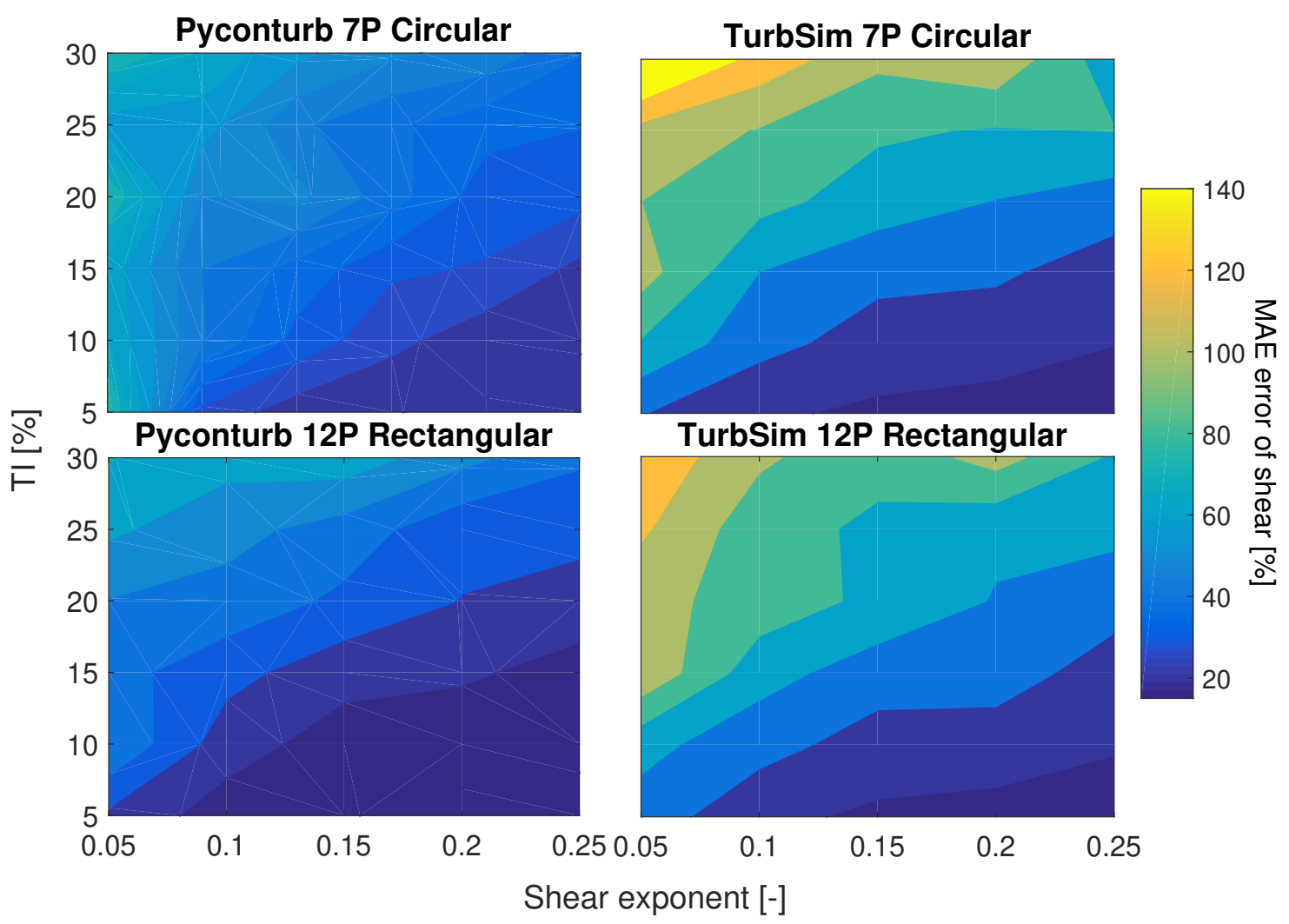

Fig. 18 MAE percentage of the shear time series error compared to the original wind field.

error is below $30 \%$ for all cases considered with a TI equal or smaller than $20 \%$. The results for the 12P pattern (not shown here) were similar with a decrease on the magnitude of error around $10 \%$ for all cases. The results suggest that, especially in low ambient turbulence situations, both methodologies and lidar setups can be considered accurate in capturing the instantaneous $U$.

The MAE percentage of the REWS time series with varying shear and TI for both codes and lidar patterns is shown in figure 20. It is seen that shear has very low influence in the error and TI is the driver of the error variation. As before increased turbulence leads to increased error. The error magnitude is significantly lower than the error seen in figure 19. Since we consider a spatially averaged quantity (REWS) the error is averaging out and decreasing in contrast to looking at the error of each point individually. The error with the PyConTurb algorithm is almost half of the error with the TurbSim algorithm. Moreover the error from PyConTurb seems to be less sensitive to the variation of TI. The overall magnitude of error is quite low in both cases reaching a maximum of $6 \%$ with PyConTurb and $12 \%$ with TurbSim. The two patterns have similar trends with the $12 \mathrm{P}$ resulting in lower error for every case. This result suggests that applications requiring instantaneous calculations of REWS such as lidar load validation would be benefited by using the 12P pattern while identifying that the main uncertainty driver is turbulence. The lack of smoothness of the contour is related to the issue discussed at the beginning of the section regarding the number of seeds considered.

\section{Discussion}

In this section we discuss some aspects of the methods and the results investigated that require further analysis. As seen from the results, the spatial coherence distribution algorithm between measured and interpolated points is the main driver for the differences in the errors between TurbSim and PyConTurb. In order to examine this behavior, we performed a study were we applied to both algorithms perfect lidar measurements taking into account initially all the grid points (1681 in total), and then every second, third and so on grid point. The $U$ time series MAE percentage per grid point is shown in figure 21 for all and every fourth point measurements. When all points are considered we see the expected behavior of zero error with PyConTurb. With TurbSim we see that the the lower right half of the plane contains 


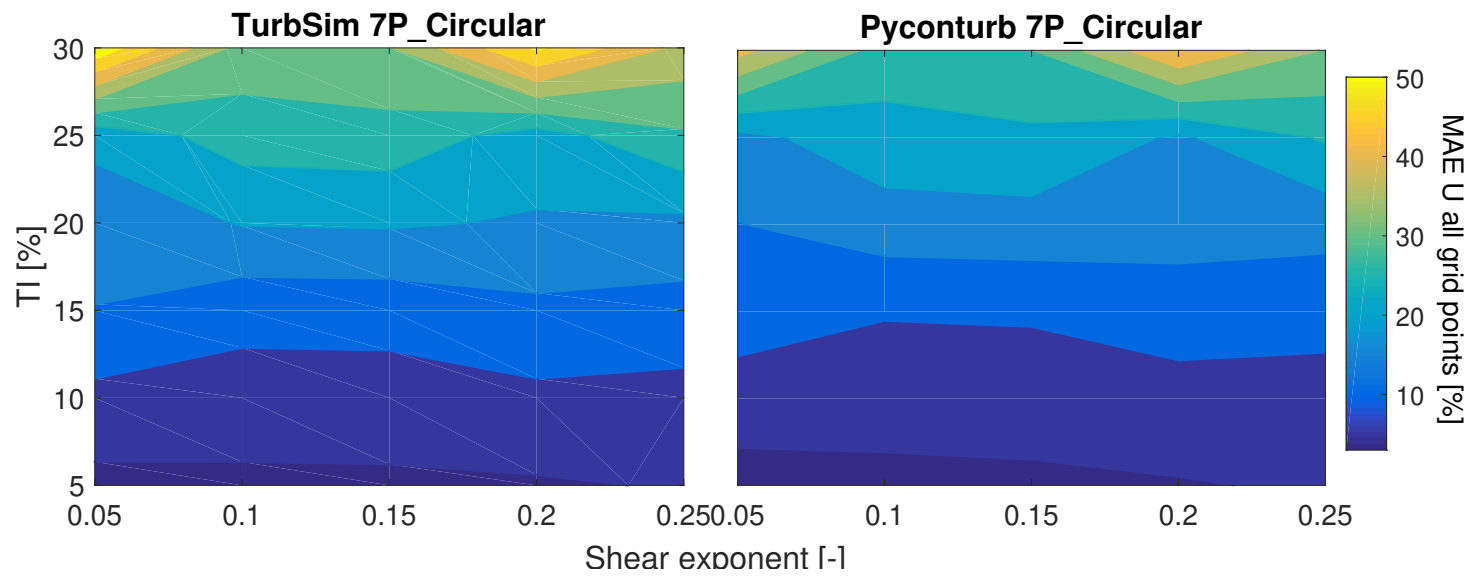

Fig. 19 MAE percentage of $U$ for the whole grid using the 7P lidar pattern and both constraining algorithms. Mean velocity is $8 \mathrm{~m} / \mathrm{s}$ and time step of measurements is $2 \mathrm{~s}$.

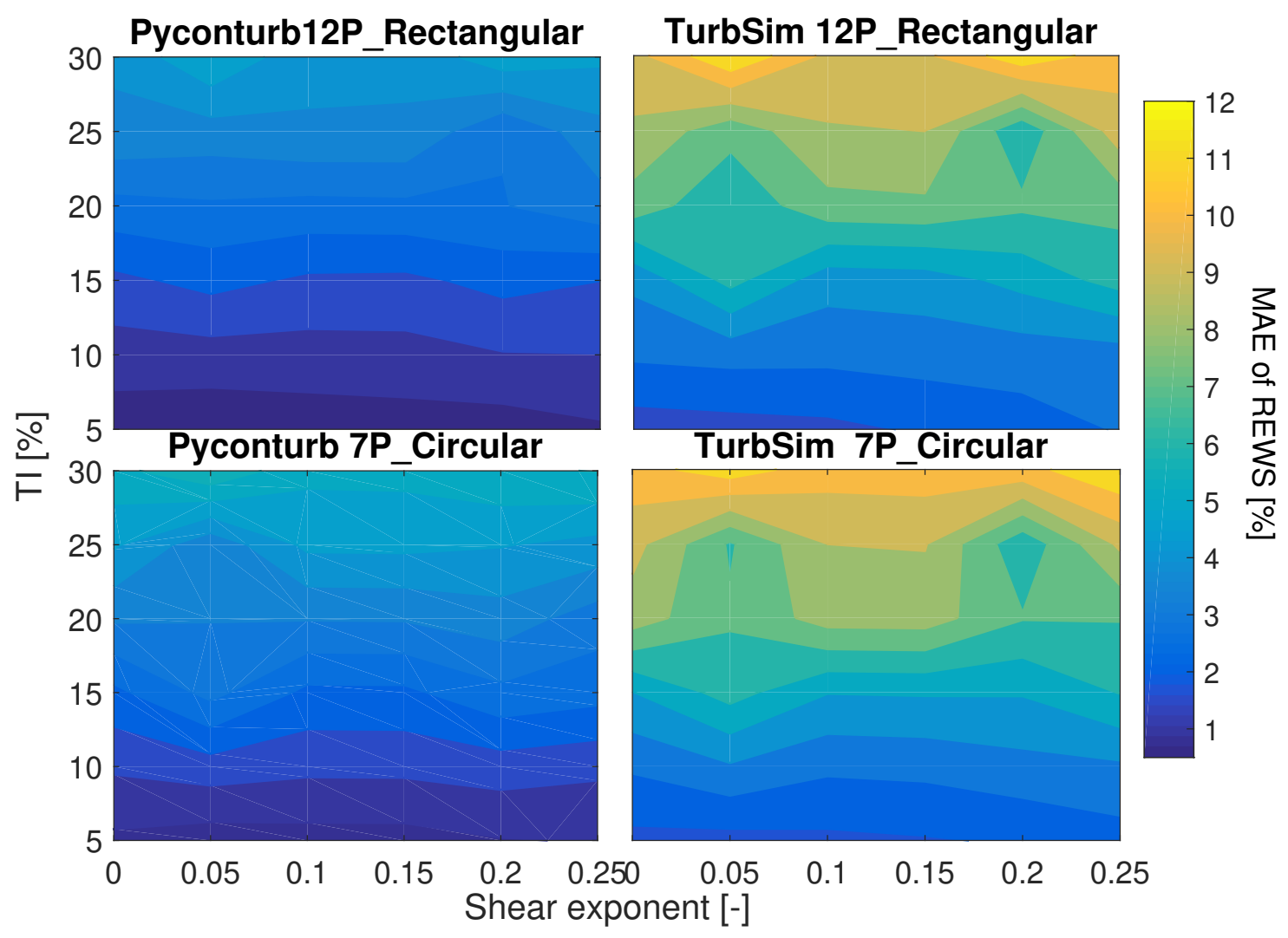

Fig. 20 Mean absolute error percentage of REWS time series using both lidar patterns and constraining algorithms. Mean velocity is $8 \mathrm{~m} / \mathrm{s}$ and time step of measurements is $2 \mathrm{~s}$.

unexpected errors up to $15 \%$. Seeing the case with every fourth point used we observe that the error in the TurbSim case is distributed more smoothly in the whole grid domain. The magnitude of error is higher in Turbsim in every case due to the spatial coherence distribution algorithm discussed earlier. The behavior of both codes when decreasing the amount of points supplied (not shown here) was as expected with the error magnitude increasing proportionally to the decrease of points. This test indicates a possible issue in the implementation of the constraining algorithm in TurbSim. 
The extent to which this affects the result when fewer points are measured, like the patterns we used in the results section, is not clear and needs to be further investigated.

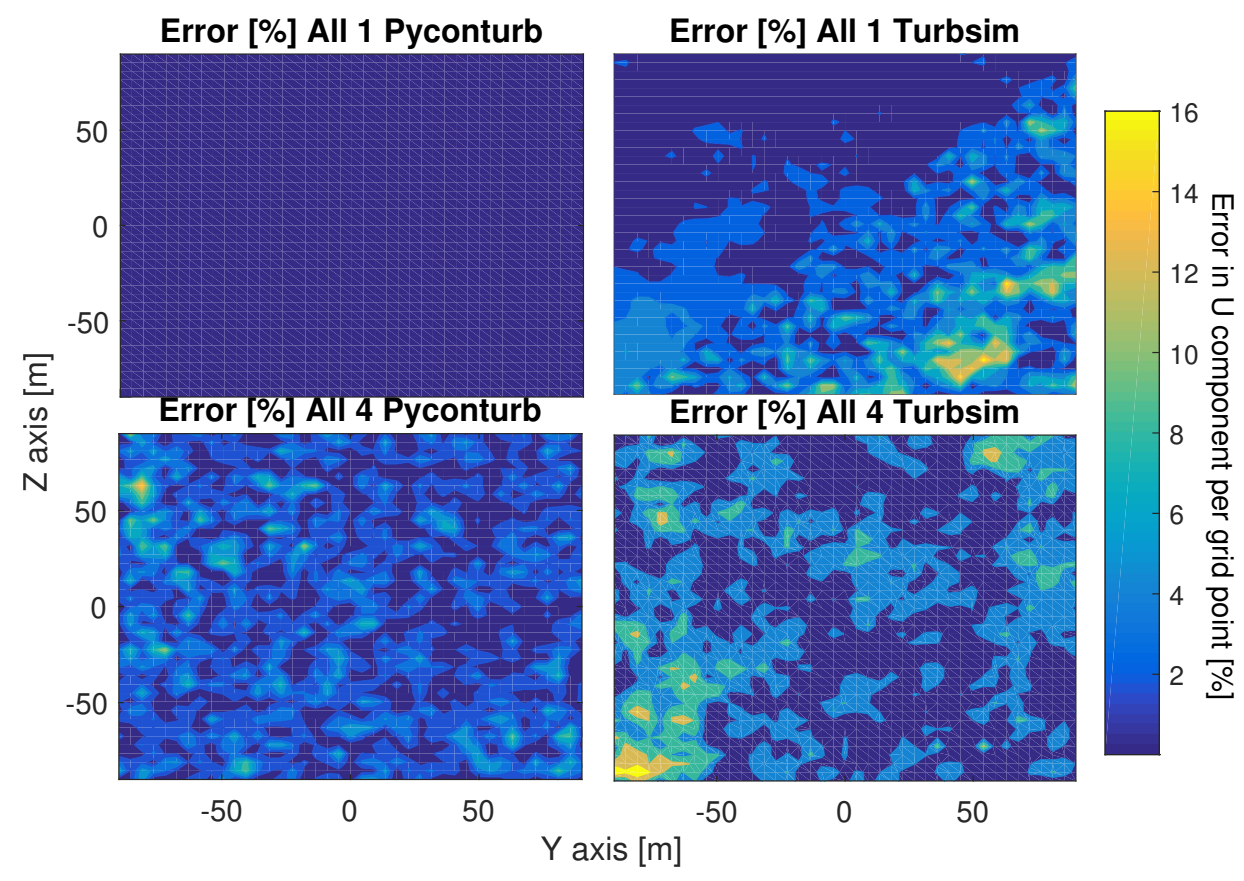

Fig. 21 Errors between original and synthetic wind fields for PyConTurb and TurbSim and using perfect lidar measurements with all and every fourth grid points used.

In section IVB we used the 7P and 12P patterns and not 5P and 9P shown in the IVA. One reason for this was the behavior of PyConTurb. In figure 22 we show a snapshot of the a wind field constrained with the 5P and 9P lidar patterns along with the original wind field. Here we can see a shortcoming of the PyConTurb implementation. The coherence of the interpolated points is defined according to the distance from the measured points. When the pattern is such that there are points in the grid that have an equal distance to the measured points, the algorithm calculates the spatial coherence based on one of the points. This leads to breaking the grid domain in smaller regions that are not smoothly correlated with each other leading to high errors. Possible workarounds for this issue could be either to apply a kind of smoothing function on the interfaces of these regions or change the way the neighborhoods of influence are calculated for each interpolated point in order to overlap them smoothly.

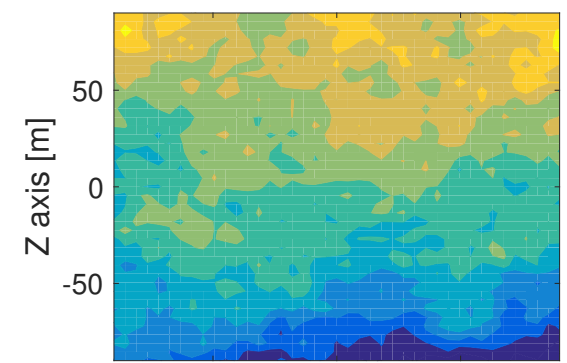

$-50$
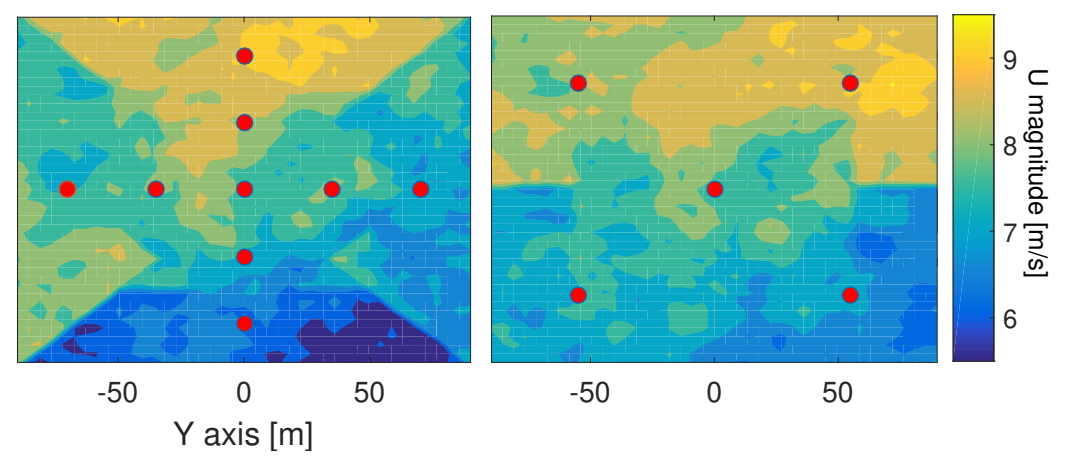

Fig. 22 Wind speed magnitude (U component) contours of one slice in time. From left to right: Original wind field, constrained wind field with PyConTurb using the 5 point rectangular pattern, and constrained wind field with PyConTurb using the 9 point cross pattern. Red circles indicate yh the locations measured by the lidar.

Another important issue for constraining synthetic wind fields with lidar measurements is the treatment of the time 
shift between lidar measurements. Unless the device has multiple synchronized beams, every measured point will come with a time shift as explained in section II. Currently, none of the algorithms can take this into account. To tackle this issue, we can on the one hand consider adjusting the algorithms so that they accept each individual lidar measurement at each exact time step, which in turn will lead to one constrained point per slice. On the other hand we can interpolate the measurements to synchronize them or average the value on one time stamp which can lead to biasing the result.

The previous discussion shows that, since both codes were developed considering met mast measurements as inputs for the constraints, some modifications are still required to accommodate the spatial variation of lidar measurements. We are currently working on modifying the algorithms to make them more suitable for lidar inputs. Both topics mentioned earlier need more investigation and are part of the future planned work.

The computational time is another point to comment on the different methodologies. Due to the difference spatial coherence implementation PyConTurb has much higher computational cost. For each of the wind fields constrained here, with a grid of $41 \times 41$ points and 900 time steps, the average time of TurbSim was 5 minutes and for PyConTurb 100 minutes. Moreover, the requirements in CPU and memory from PyConTurb made it infeasible to run the simulation in a normal laptop and limited the parallel executions on a computational cluster. This can become important when batch processing is required or optimization studies are considered.

Moreover, it is worth mentioning the effect of the turbulent box discretization. In order to obtain realistic virtual lidar measurements the grid has to be dense enough for the application. As observed in a short convergence study, as the discretization gets coarser the effects of volume averaging combined with LOS reconstruction and noise are diminishing. As the grid becomes denser there is a limit above which the results converge. Increasing the grid density has a trade off with computational cost. To conclude, depending on the application one should be aware that the original wind field discretization can possibly bias the results and should be taken into account.

As mentioned in previous sections, all the studies shown here considered the lidar being perfectly aligned with the wind field. This reduces significantly the uncertainty coming from the wind field reconstruction methods. In real measurements the wind direction changes in a continuous manner with a frequency higher than frequency with which the turbine yaws. Currently ViConDAR cannot capture this effect as we can only impose a constant flow misalignment. The virtual measurement can become more realistic if we impose a dynamic flow misalignment. For this we consider deriving from measurements some average frequency spectrum of wind direction and implement as a random-phase initiated Gaussian time realization along our measurements.

Finally, it is important to check the correlation of current results to load response of the turbine when considering the load validation using lidar measurements. The metrics were chosen in order to quantify the level of the agreement of the constrained wind fields to the original ones. It is important to investigate how these discrepancies affect the aeroelastic response of the turbine. Based on the individual dimensions, aerodynamic configuration and control algorithm each individual turbine will respond differently to the wind excitation. Hence, we need to identify the correlation between wind field and load discrepancies. Moreover, the present results showed that the sampling rate of the lidar is not a major driver of uncertainty for most of the examined metrics. This is expected to have much higher influence when loads are considered. To sum up, closing the loop of the suggested methodology with load simulations will give a much clearer picture of the real drivers of uncertainty for load validation using lidar measurements.

\section{Conclusions}

This work introduces ViConDAR, an open source modular framework for lidar related applications in wind energy allowing experience and knowledge to be shared across the community. The structure of the framework along with the underlying models and assumptions is described. The tool is capable of simulating realistic lidar measurements from a synthetic wind field and feeding them as inputs to constrained turbulence generators, namely TurbSim and PyConTurb. The outputs can be used as inputs to aeroelastic simulations. The tool offers also post processing capabilities which, along with the modular structure, makes it versatile for different use cases. It can be used as a common ground for researchers to evaluate novel methods. Additionally, ViConDAR can be utilized to identify optimal desired parameters of a lidar for a particular application such as lidar load validation or lidar assisted control. Moreover, it can be used to optimize trajectories and other parameters of a specific device according to the application.

In the results we demonstrated two applications of the framework. In the first we compared lidar measurements with different scanning patterns and sampling rates under varying atmospheric conditions to the reference wind field. The main driver of uncertainty in most cases was turbulence. The best agreement was under conditions with low turbulence and high shear. The effect of sampling rate was found to be very weak and increased the error only in very low sampling rates. From the limited range of lidar scanning patterns tested, the seven point circular was found to be the one showing 
overall the lowest error. In the second application we performed a sensitivity analysis of the convergence between the original and the constrained wind field, using different constraining methodologies under varying atmospheric conditions. Various error metrics were used to identify the convergence in time and space. The results showed the importance of taking into account the two dimensional spatial correlation of the constraints to the interpolated points. In most cases the error magnitude was lower when PyConTurb was used. The mean quantities showed a low error in agreement with the relevant literature while the instantaneous quantities showed higher errors.

The overall effectiveness of the suggested framework can be validated in a numerical level by comparing the simulated load response between the original and the constrained wind field. In an experimental level this can be done with load measurement campaigns where the measured loads are compared with the loads obtained with lidar setups optimized with the framework. Both tasks are planned for the near future. In addition some limitations of current algorithms for generating constrained turbulence using lidar measurements due to the spatial and temporal discretization of the measurements were identified and possible counter measures were discussed, which are expected to be tested in future work.

\section{Acknowledgments}

This study was funded by the German Federal Ministry for Economic Affairs and Energy (BMWi) in the framework of the national joint research project RAVE - OWP Control (ref, 0324131B) and is part of the research done in the WindForS wind energy research cluster.

\section{References}

[1] Clive, P. J. M., "Lidar and resource assessment for wind power applications: the state of the art," 2008 , p. 711107. doi:10.1117/12.800284.

[2] Borraccino, A., "Remotely measuring the wind using turbine-mounted lidars: Application to power performance testing," Ph.D. thesis, DTU, Denmark, 2017. doi:10.11581/DTU:00000021.

[3] Schlipf, D., "Lidar-assisted control concepts for wind turbines," Phd thesis, Stuttgart University, 2016. doi:doi.org/10.18419/ OPUS-8796.

[4] Dimitrov, N., Borraccino, A., Peña, A., Natarajan, A., and Mann, J., "Wind turbine load validation using lidar-based wind retrievals," Wind Energy, Vol. 22, No. 11, 2019, pp. 1512-1533. doi:10.1002/we.2385.

[5] Clifton, A., Clive, P., Gottschall, J., Schlipf, D., Simley, E., Simmons, L., Stein, D., Trabucchi, D., Vasiljevic, N., and Würth, I., "IEA Wind Task 32: Wind Lidar Identifying and Mitigating Barriers to the Adoption of Wind Lidar," Remote Sensing, Vol. 10, No. 3, 2018, p. 406. doi:10.3390/rs10030406.

[6] Jonkman, B. J., “TurbSim User's Guide v2. 00.00,” Tech. Rep. October 2014, NREL, 2014. URL https://nwtc.nrel .gov/ TurbSim.

[7] Rinker, J. M., "PyConTurb: an open-source constrained turbulence generator," Journal of Physics: Conference Series, Vol. 1037, 2018, p. 062032. doi:10.1088/1742-6596/1037/6/062032.

[8] Veers, P. S., “Three-Dimensional Wind Simulation,SAND88-0152,” Tech. rep., SANDIA, SAND88-0152, 1988.

[9] Mann, J., "Wind field simulation,” Probabilistic Engineering Mechanics, Vol. 13, No. 4, 1998, pp. 269-282. doi:10.1016/S02668920(97)00036-2.

[10] Jonkman, J., and Marshall, B., “FAST User's Guide,” Tech. Rep. 6, 2005. doi:10.2172/15020796, URL http: //www . ncbi . nlm.nih.gov/pubmed/21564034.

[11] International Electrotechnical Commision (IEC), "Wind turbines Part 1: Design requirements 61400-1, 3rd edition," Tech. rep., International Electrotechnical Commision (IEC), Switzerland, 2005.

[12] Bierbooms, W., and Veldkamp, D., "Time Domain Comparison of Simulated and Measured Wind Turbine Loads Using Constrained Wind Fields," Wind Energy, Springer Berlin Heidelberg, Berlin, Heidelberg, 2007, pp. 15-20. doi:10.1007/978-3540-33866-6_3.

[13] Schlipf, D., and Raach, S., “Turbulent Extreme Event Simulations for Lidar-Assisted Wind Turbine Control," Journal of Physics: Conference Series, Vol. 753, No. 5, 2016. doi:10.1088/1742-6596/753/5/052011. 
[14] Dimitrov, N., and Natarajan, A., "Application of simulated lidar scanning patterns to constrained Gaussian turbulence fields for load validation,” Wind Energy, Vol. 20, No. 1, 2017, pp. 79-95. doi:10.1002/we.1992.

[15] Nielsen, M., Larsen, G., Mann, J., Ott, S., Hansen, K. S., and Pedersen, B. J., Wind simulation for extreme and fatigue loads, Vol. 1437, DTU, 2003.

[16] Hoffman, Y., and Ribak, E., "Constrained realizations of Gaussian fields - A simple algorithm,” The Astrophysical Journal, Vol. 380, No. 1987, 1991, p. L5. doi:10.1086/186160.

[17] Kelley, N. D., “Turbulence-Turbine Interaction: The Basis for the Development of the TurbSim Stochastic Simulator,” Tech. Rep. November, National Renewable Energy Laboratory (NREL), Golden, CO (United States), nov 2011. doi:10.2172/1031981.

[18] Goit, J. P., Shimada, S., and Kogaki, T., “Can LiDARs Replace Meteorological Masts in Wind Energy?” Energies, Vol. 12, No. 19, 2019, p. 3680. doi:10.3390/en12193680.

[19] Bak, C., Zahle, F., Bitsche, R., Yde, A., Henriksen, L. C., Nata, A., and Hansen, M. H., "Description of the DTU 10 MW Reference Wind Turbine,” Tech. Rep. July, DTU, Roskilde, 2013. URL https: //dtu-10mw-rwt.vindenergi .dtu.dk. 